\title{
Lifetime measurements and oscillator strengths in singly ionized scandium and the solar abundance of scandium
}

\author{
A. Pehlivan Rhodin,,${ }^{1,2 \star}$ M. T. Belmonte,${ }^{3}$ L. Engström, ${ }^{4}$ H. Lundberg, ${ }^{4}$ H. Nilsson, ${ }^{1}$ \\ H. Hartman, ${ }^{1,2}$ J. C. Pickering, ${ }^{3}$ C. Clear, ${ }^{3}$ P. Quinet, ${ }^{5,6}$ V. Fivet ${ }^{5}$ and P. Palmeri ${ }^{5 \star}$ \\ ${ }^{1}$ Lund Observatory, Lund University, PO Box 43, SE-221 00 Lund, Sweden \\ ${ }^{2}$ Materials Science and Applied Mathematics, Malmö University, SE-205 06 Malmö, Sweden \\ ${ }^{3}$ Physics Department, Blackett Laboratory, Imperial College London, London SW7 2BZ, UK \\ ${ }^{4}$ Department of Physics, Lund Institute of Technology, PO Box 118, SE-221 00 Lund, Sweden \\ ${ }^{5}$ Physique Atomique et Astrophysique, Université de Mons-UMONS, 20 Place du Parc, B-7000 Mons, Belgium \\ ${ }^{6}$ IPNAS, Université de Liège, B15 Sart Tilman, B-4000 Liège, Belgium
}

Accepted 2017 August 18. Received 2017 August 18; in original form 2017 July 12

\begin{abstract}
The lifetimes of 17 even-parity levels $\left(3 \mathrm{~d} 5 \mathrm{~s}, 3 \mathrm{~d} 4 \mathrm{~d}, 3 \mathrm{~d} 6 \mathrm{~s}\right.$ and $\left.4 \mathrm{p}^{2}\right)$ in the region $57743-77837 \mathrm{~cm}^{-1}$ of singly ionized scandium (Sc II) were measured by two-step timeresolved laser induced fluorescence spectroscopy. Oscillator strengths of 57 lines from these highly excited upper levels were derived using a hollow cathode discharge lamp and a Fourier transform spectrometer. In addition, Hartree-Fock calculations where both the main relativistic and core-polarization effects were taken into account were carried out for both low- and high-excitation levels. There is a good agreement for most of the lines between our calculated branching fractions and the measurements of Lawler \& Dakin in the region 9000-45000 $\mathrm{cm}^{-1}$ for low excitation levels and with our measurements for high excitation levels in the region $23500-63100 \mathrm{~cm}^{-1}$. This, in turn, allowed us to combine the calculated branching fractions with the available experimental lifetimes to determine semi-empirical oscillator strengths for a set of $380 \mathrm{E} 1$ transitions in Sc II. These oscillator strengths include the weak lines that were used previously to derive the solar abundance of scandium. The solar abundance of scandium is now estimated to $\log \epsilon_{\odot}=3.04 \pm 0.13$ using these semi-empirical oscillator strengths to shift the values determined by Scott et al. The new estimated abundance value is in agreement with the meteoritic value $\left(\log \epsilon_{\text {met }}=3.05 \pm 0.02\right)$ of Lodders, Palme \& Gail.
\end{abstract}

Key words: atomic data-methods: laboratory: atomic-methods: numerical-techniques: spectroscopic-Sun: abundances.

\section{INTRODUCTION}

The iron-group elements $(21 \leq Z \leq 28)$ are produced during supernova type Ia explosions, while supernova type II explosions are responsible for the formation of $\alpha$-elements such as $\mathrm{Mg}, \mathrm{Si}$ and $\mathrm{S}$. The even- $Z$ nuclei such as $\mathrm{S}, \mathrm{Ca}, \mathrm{Ti}, \mathrm{Cr}$ and $\mathrm{Fe}$ have higher cosmic abundance compared to the odd- $Z$ nuclei located in between because of the consecutive capture of $\alpha$-particles. The production of odd- $Z$ elements is not well understood and does not follow the abundance trends of the $\alpha$-elements, indicating non-common production mechanisms. In recent years, this has caused an increasing interest in the odd- $Z$ iron-peak elements in astrophysics. Abundance determinations in stars constrain the stellar evolution and supernova explosion models (Pagel 2009). Moreover, transitions from highly excited levels have an additional diagnostic value since they can be used to

^E-mail: asli.pehlivan@mah.se (APR); patrick.palmeri@umons.ac.be (PP) benchmark non-local thermodynamical equilibrium (NLTE) modelling of stellar atmospheres. Besides the development of 3D hydrodynamic model atmospheres, a trustworthy NLTE treatment is the current challenge for accurate stellar abundances. High-precision atomic data for selected lines are important for this development (Lind, Bergmann \& Asplund 2012).

In the case of scandium $(Z=21)$, a realistic 3D NLTE solar atmosphere model has been used by Scott et al. (2015) to revise the solar abundance of scandium resulting in a photospheric value in significant disagreement with the meteoritic abundance (Lodders et al. 2009). Scott et al. (2015) used experimental transition probabilities of five Sc I and nine Sc II lines determined by Lawler \& Dakin (1989). The latter authors combined their measured branching fractions with the time-resolved laser induced fluorescence (TR-LIF) lifetimes of Marsden et al. (1988) to obtain absolute $A$-values for transitions depopulating 51 levels in Sc I and 18 levels in Sc II. In Marsden et al. (1988), only three highly excited evenparity levels of Sc II, belonging to $3 \mathrm{~d}_{4} \mathrm{~d}^{3} \mathrm{G}$, were measured. Older 
lifetime measurements in singly ionized scandium have focused on lower excited odd-parity 3d4p and 4s4p levels (Buchta et al. 1971; Arnesen et al. 1976; Palenius, Curtis \& Lundlin 1976; Vogel et al. 1985). On the theoretical side, the most recent calculations of E1 oscillator strengths in Sc II are given in Ruczkowski, Elantkowska \& Dembczynski (2014) and Kurucz (2011).

The main goal of this work is to provide a new set of experimental $f$-values for transitions depopulating the highly excited even-parity levels in Sc II, and new calculations for both low- and high-excitation levels and lines. Descriptions of our measurements are presented in Sections 2 and 3. The theoretical method used for the calculation of the radiative parameters is described in Section 4. In Section 5, our results are presented and compared to data available in the literature. The consequence of the proposed set of oscillator strengths on the solar abundance of scandium is discussed in Section 6. Finally, our conclusions are given in Section 7.

\section{LIFETIME MEASUREMENTS}

The experimental set-up for the two-step Time-Resolved Laser Induced Fluorescence (TR-LIF) measurements at the Lund High Power Laser Facility has been described in detail by Engström et al. (2014) and Lundberg et al. (2016). For an overview, we refer to fig. 1 in Lundberg et al. (2016), and here we give only the most important details. A frequency doubled Nd:YAG laser (Continuum Surelite) with $10 \mathrm{~ns}$ pulses was used to produce the free scandium ions by focusing the light on a rotating solid scandium sample in a vacuum chamber with a pressure of around $10^{-4}$ mbar. The ions in the plasma cone were crossed by two laser beams, a few millimetre above the solid sample, generating the two-step excitations. The fluorescence signal was detected in a direction perpendicular to both the ablation and excitation lasers.

For the first step (4s-4p), we used a Continuum Nd-60 dye laser with either DCM or Pyridine 2 dyes. The 10 ns long pulses were frequency doubled using a KDP crystal, giving the wavelengths needed for the first step. The second laser system excited the final high-energy levels. It consists of a frequency doubled Continuum NY-82 Nd:YAG laser pumping a Continuum Nd-60 dye laser with either DCM or Oxacin dye for wavelengths below or above $660 \mathrm{~nm}$, respectively. The pulse length was reduced from 10 ns to less than $1 \mathrm{~ns}$ by stimulated Brillouin scattering. The output was frequency doubled using a KDP crystal and, where higher energy was needed, tripled with a BBO crystal.

For two-step excitation, the timing between the pulses is crucial. For this purpose, a delay generator ensures that the second step is timed to when the population of the intermediate state is at its flat maximum as determined by observing the decay of this level in another channel, see fig. 2 in Lundberg et al. (2016).

The fluorescence emitted by the scandium ions was filtered by a $1 / 8 \mathrm{~m}$ grating monochromator with its $0.28 \mathrm{~mm}$ wide entrance slit oriented parallel to the excitation laser beams. This fluorescence light was recorded using a fast micro-channel-plate photomultiplier tube (Hamamatsu R3809U) and digitized using a Tektronix DPO 7254 oscilloscope with $2.5 \mathrm{GHz}$ analogue bandwidth. We used the second spectral order with a $0.5 \mathrm{~nm}$ observed line width for all measurements. The excitation laser pulse shape was recorded simultaneously using a fast photo diode and digitized by another channel of the oscilloscope. All decay curves were averaged over 1000 laser pulses and analysed using the DECFIT software (Palmeri et al. 2008) by fitting a single exponential function convoluted by the measured shape of the second-step laser pulse and a background function to the observed decay.

The excitation schemes of the measured $\mathrm{Sc}$ II levels are presented in Table 1. This table shows the intermediate levels and their excitation wavelengths, the final levels and their excitation wavelengths from the intermediate levels together with the detection channel level and wavelength. For the levels $4 \mathrm{~d}^{3} \mathrm{~S}_{1}, 4 \mathrm{~d}{ }^{1} \mathrm{D}_{2}$ and $4 \mathrm{p}^{2}{ }^{3} \mathrm{P}_{2}$, it was possible to record the decay in more than one channel. We did not find any differences in the lifetimes obtained from the different channels. Sc II is a complex spectrum with a dense level structure, as shown in Fig. 1. Line blending can be caused by cascades or fluorescence from the intermediate level as discussed by Lundberg et al. (2016). For all measurements, we investigated if there was a line blend affecting the recorded curves. Due to the small spectral width of the laser compared to the energy level separations, we avoid exciting multiple levels.

To investigate any possible saturation effects in the second-step excitation, a set of neutral density filters was placed in the excitation beam. The delay between the ablation and first excitation pulse, the geometrical alignment of the lasers with respect to the target as well as the intensity of the ablation laser were varied to test time-of-flight effects. No systematic effects were observed.

As discussed in Palmeri et al. (2008), the weighting of individual data points, hence the purely statistical uncertainty in the fitted lifetime, is difficult to estimate accurately because the digitizing process is not strictly a counting measurement. However, extensive tests have shown that even for weak lines the dominating factor is the variation between different measurements. The uncertainty in Table 2 represents the uncertainty of 10-20 measurements performed over several days. The difference between subsequent curves is significantly lower than the quoted uncertainty, usually less than 1 per cent.

\section{BRANCHING FRACTION MEASUREMENTS}

A water-cooled hollow cathode discharge lamp (HCL) was used to produce the free scandium ions. The lamp has an iron cathode with anodes on each side, separated by glass cylinders. A small piece of scandium was placed in the cathode. We used argon, with a pressure of 0.3 Torr, as a buffer gas and applied currents ranging from 0.2 to $0.5 \mathrm{~A}$. These measurements at different currents are very important to find and compensate for self-absorption effects. If self-absorption is not treated correctly, the measured relative line intensity may be less than the true intensity of the line. This in turn changes the branching fraction that is essential to derive oscillator strengths. Self-absorption was observed in the case of the $3 \mathrm{~d}_{4} \mathrm{~d}^{3} \mathrm{D}_{3}$, $3 \mathrm{~d} 4 \mathrm{~d}^{3} \mathrm{~S}_{1}$ and $3 \mathrm{~d} 4 \mathrm{~d}^{3} \mathrm{P}_{2}$ levels, and the affected lines were corrected. More details on this procedure can be found in Pehlivan, Nilsson \& Hartman (2015).

The spectra were recorded with the vacuum ultraviolet Fourier transform spectrometer (VUV FTS) at the Blackett Laboratory, Imperial College London (Pickering 2002) in the interval 23500 $63100 \mathrm{~cm}^{-1}(425-158 \mathrm{~nm})$ using a resolution of $0.039 \mathrm{~cm}^{-1}$. We used two different photomultiplier tube detectors: Hamamatsu R7154 and R11568, the latter with a UG5 filter. Each scandium measurement consists of 12 co-added scans. To determine the relative response functions of the system, we used standard lamps: a tungsten filament lamp (800-300 nm) and a deuterium lamp (410-116 nm) for the wavelength region $(425-210 \mathrm{~nm})$, and a deuterium standard lamp alone for the region $(317-158 \mathrm{~nm})$. The tungsten lamp was calibrated by the UK National Physical Laboratory 
Table 1. Measured Sc II levels and the corresponding two-step excitation schemes.

\begin{tabular}{|c|c|c|c|c|c|c|c|c|}
\hline \multirow{2}{*}{$\begin{array}{l}\text { Final } \\
\text { level }^{a}\end{array}$} & \multicolumn{3}{|c|}{ First-step excitation } & \multicolumn{3}{|c|}{ Second-step excitation } & \multicolumn{2}{|c|}{ Detection } \\
\hline & $\begin{array}{c}\text { Starting } \\
\text { level }^{a}\left(\mathrm{~cm}^{-1}\right)\end{array}$ & $\begin{array}{l}\text { Intermediate } \\
\text { level }{ }^{a}\left(\mathrm{~cm}^{-1}\right)\end{array}$ & $\begin{array}{l}\lambda_{\text {air }} \\
(\mathrm{nm})\end{array}$ & $\begin{array}{l}\text { Final level }^{a} \\
\left(\mathrm{~cm}^{-1}\right)\end{array}$ & $\begin{array}{l}\lambda_{\text {air }} \\
(\mathrm{nm})\end{array}$ & Scheme $^{b}$ & $\begin{array}{l}\text { Lower level }^{a} \\
\left(\mathrm{~cm}^{-1}\right)\end{array}$ & $\begin{array}{l}\lambda_{\text {air }} \\
(\mathrm{nm})\end{array}$ \\
\hline $5 \mathrm{~s}^{3} \mathrm{D}_{3}$ & 67.72 & 27602.45 & 363.07 & 57743.92 & 331.67 & $2 \omega$ & 27841.35 & 334.32 \\
\hline $5 \mathrm{~s}^{1} \mathrm{D}_{2}$ & 67.72 & 27602.45 & 363.07 & 58252.09 & 326.17 & $2 \omega$ & 32349.98 & 385.96 \\
\hline $4 d^{1} F_{3}$ & 177.76 & 29823.93 & 337.22 & 59528.42 & 336.55 & $2 \omega$ & 26081.34 & 298.89 \\
\hline $4 d^{3} D_{1}$ & 177.76 & 29823.93 & 337.22 & 59875.08 & 332.67 & $2 \omega$ & 27917.78 & 312.83 \\
\hline $4 d^{3} D_{2}$ & 177.76 & 29823.93 & 337.22 & 59929.46 & 332.07 & $2 \omega$ & 28021.29 & 313.31 \\
\hline $4 d^{3} D_{3}$ & 177.76 & 29823.93 & 337.22 & 60001.91 & 331.27 & $2 \omega$ & 28161.17 & 313.97 \\
\hline $4 d^{3} G_{3}$ & 177.76 & 29823.93 & 337.22 & 60267.16 & 328.39 & $2 \omega$ & 27443.71 & 304.57 \\
\hline $4 d^{1} P_{1}$ & 177.76 & 29823.93 & 337.22 & 60400.41 & 326.95 & $2 \omega$ & 26081.34 & 291.30 \\
\hline $4 d^{3} S_{1}$ & 177.76 & 29823.93 & 337.22 & 61071.43 & 319.93 & $2 \omega$ & $\begin{array}{l}29823.93 \\
39345.52\end{array}$ & $\begin{array}{l}319.93 \\
460.15\end{array}$ \\
\hline $4 d^{3} F_{2}$ & 2540.95 & 32349.98 & 335.37 & 63374.63 & 322.23 & $2 \omega$ & 27917.78 & 281.95 \\
\hline $4 d^{3} F_{4}$ & 2540.95 & 32349.98 & 335.37 & 63528.54 & 320.64 & $2 \omega$ & 28161.17 & 282.66 \\
\hline $4 d^{1} D_{2}$ & 2540.95 & 32349.98 & 335.37 & 64366.68 & 312.25 & $2 \omega$ & $\begin{array}{l}26081.34 \\
30815.70\end{array}$ & $\begin{array}{l}261.12 \\
297.97\end{array}$ \\
\hline $4 d^{3} P_{2}$ & 2540.95 & 32349.98 & 335.37 & 64705.89 & 308.98 & $2 \omega$ & 29823.93 & 286.60 \\
\hline $4 p^{2}{ }^{1} D_{2}$ & 177.76 & 28161.17 & 357.25 & 74433.30 & 216.04 & $3 \omega$ & 32349.98 & 237.55 \\
\hline $4 p^{2}{ }^{3} \mathrm{P}_{1}$ & 177.76 & 29823.93 & 337.22 & 76360.80 & 214.82 & $3 \omega$ & 39345.52 & 270.08 \\
\hline $4 \mathrm{p}^{2}{ }^{3} \mathrm{P}_{2}$ & 177.76 & 29823.93 & 337.22 & 76589.30 & 213.76 & $3 \omega$ & $\begin{array}{l}28161.17 \\
39115.04 \\
39345.52\end{array}$ & $\begin{array}{l}206.43 \\
266.77 \\
268.42\end{array}$ \\
\hline $6 \mathrm{~s}^{3} \mathrm{D}_{3}$ & 177.76 & 29823.93 & 337.22 & 77387.17 & 210.18 & $3 \omega$ & 28161.17 & 203.08 \\
\hline
\end{tabular}

Notes. ${ }^{a}$ All energy-level values and wavelength values are from Johansson \& Litzén (1980).

${ }^{b} 2 \omega$ and $3 \omega$ stand for, respectively, frequency doubling and tripling excitation schemes. All first-step levels are excited using a frequency doubling scheme $(2 \omega)$.

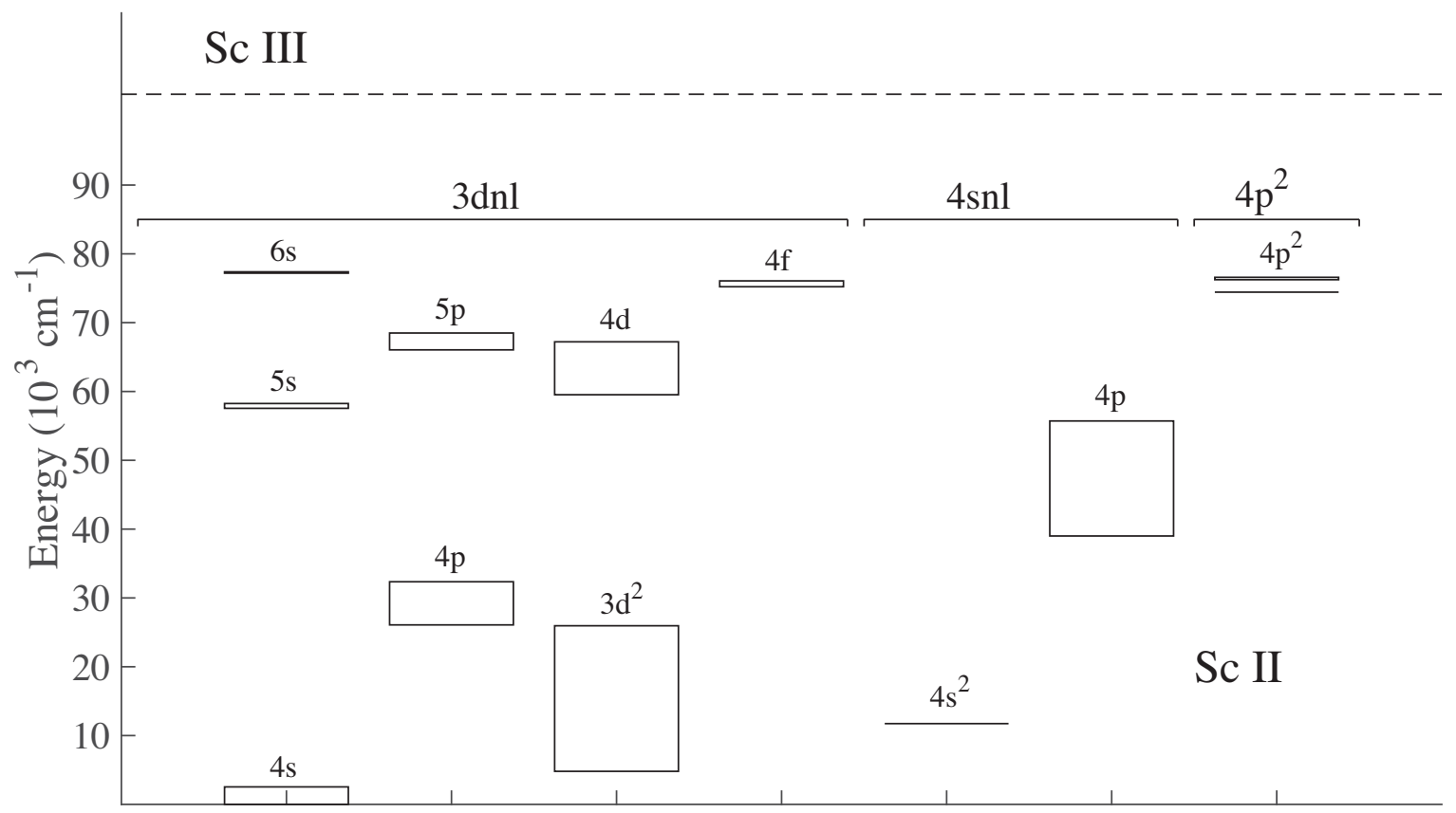

Figure 1. Partial energy level diagram of Sc II, the energy level values are from Johansson \& Litzén (1980). Each box consists of several levels.

and the deuterium lamp by Physikalisch-Technische Bundesanstalt, in Berlin. In the region where the lamps overlap, the response functions were placed on the same relative scale. We recorded the spectrum of the calibration lamps immediately before and after each scandium measurement. The HCL and the calibration lamps were placed at the same distances from the FTS, and a mirror was used to select the light source without moving the lamps.
In astrophysics, oscillator strengths ( $f$-values) or $\log (g f)$ values are the parameters used for abundance analysis. The $f$-value is proportional to the transition probability for E1 transitions by

$f=\frac{g_{\mathrm{u}}}{g_{1}} \lambda^{2} A_{\mathrm{ul}} 1.499 \times 10^{-16}$ 
Table 2. A comparison of radiative lifetimes $(\tau)$ in Sc II.

\begin{tabular}{|c|c|c|c|c|c|}
\hline Level $^{a}$ & $\begin{array}{c}E^{a} \\
\left(\mathrm{~cm}^{-1}\right)\end{array}$ & $\begin{array}{c}\tau_{\text {this cal }}^{b} \\
\text { (ns) }\end{array}$ & $\begin{array}{l}\tau_{\text {this exp }}^{c} \\
\text { (ns) }\end{array}$ & $\begin{array}{c}\tau_{\text {other exp }} \\
(\mathrm{ns})\end{array}$ & $\begin{array}{c}\tau_{\text {other cal }} \\
\text { (ns) }\end{array}$ \\
\hline $3 \mathrm{~d} 4 \mathrm{p}^{1} \mathrm{D}_{2}^{\mathrm{o}}$ & 26081.34 & 6.65 & & $\begin{array}{c}7.5 \pm 0.4^{d} \\
7.16 \pm 0.18^{e} \\
7.8 \pm 0.8^{h}\end{array}$ & $\begin{array}{l}6.54^{i} \\
7.79^{j}\end{array}$ \\
\hline $3 \mathrm{~d} 4 \mathrm{p}^{3} \mathrm{~F}_{2}^{\mathrm{o}}$ & 27443.71 & 5.68 & & $\begin{array}{l}6.2 \pm 0.3^{d} \\
6.2 \pm 0.2^{f} \\
6.5 \pm 0.4^{g}\end{array}$ & $\begin{array}{l}5.38^{i} \\
5.90^{j}\end{array}$ \\
\hline $3 \mathrm{~d} 4 \mathrm{p}^{3} \mathrm{~F}_{3}^{\mathrm{o}}$ & 27602.45 & 5.62 & & $6.1 \pm 0.3^{d}$ & $\begin{array}{l}5.32^{i} \\
5.83^{j}\end{array}$ \\
\hline $3 \mathrm{~d} 4 \mathrm{p}^{3} \mathrm{~F}_{4}^{\mathrm{o}}$ & 27841.35 & 5.54 & & $\begin{array}{l}6.1 \pm 0.3^{d} \\
5.6 \pm 0.6^{h}\end{array}$ & $\begin{array}{l}5.24^{i} \\
5.75^{j}\end{array}$ \\
\hline $3 \mathrm{~d} 4 \mathrm{p}^{3} \mathrm{D}_{1}^{\mathrm{o}}$ & 27917.78 & 4.44 & & $\begin{array}{c}4.7 \pm 0.2^{d} \\
4.61 \pm 0.10^{e}\end{array}$ & $\begin{array}{l}4.20^{i} \\
4.67^{j}\end{array}$ \\
\hline $3 \mathrm{~d} 4 \mathrm{p}^{3} \mathrm{D}_{2}^{\mathrm{o}}$ & 28021.29 & 4.41 & & $\begin{array}{c}4.7 \pm 0.2^{d} \\
4.66 \pm 0.14^{e}\end{array}$ & $\begin{array}{l}4.17^{i} \\
4.64^{j}\end{array}$ \\
\hline $3 \mathrm{~d} 4 \mathrm{p}^{3} \mathrm{D}_{3}^{\mathrm{o}}$ & 28161.17 & 4.38 & & $\begin{array}{c}4.7 \pm 0.2^{d} \\
4.55 \pm 0.15^{e} \\
6.1 \pm 0.6^{h}\end{array}$ & $\begin{array}{l}4.15^{i} \\
4.59^{j}\end{array}$ \\
\hline $3 \mathrm{~d} 4 \mathrm{p}^{3} \mathrm{P}_{0}^{\mathrm{o}}$ & 29736.27 & 6.36 & & $\begin{aligned} 7.7 & \pm 0.4^{d} \\
7.48 & \pm 0.18^{e}\end{aligned}$ & $\begin{array}{l}6.80^{i} \\
7.44^{j}\end{array}$ \\
\hline $3 \mathrm{~d} 4 \mathrm{p}^{3} \mathrm{P}_{1}^{\mathrm{o}}$ & 29742.16 & 6.39 & & $\begin{array}{l}7.6 \pm 0.4^{d} \\
7.3 \pm 0.3^{e}\end{array}$ & $\begin{array}{l}6.76^{i} \\
7.45^{j}\end{array}$ \\
\hline $3 \mathrm{~d} 4 \mathrm{p}^{3} \mathrm{P}_{2}^{\mathrm{o}}$ & 29823.93 & 6.30 & & $\begin{aligned} 7.4 & \pm 0.4^{d} \\
7.30 & \pm 0.16^{e}\end{aligned}$ & $\begin{array}{l}6.67^{i} \\
7.50^{j}\end{array}$ \\
\hline $3 \mathrm{~d} 4 \mathrm{p}^{1} \mathrm{P}_{1}^{\mathrm{o}}$ & 30815.70 & 8.10 & & $\begin{array}{l}8.8 \pm 0.4^{d} \\
8.5 \pm 0.6^{g} \\
5.5 \pm 0.5^{h}\end{array}$ & $\begin{array}{l}7.35^{i} \\
8.76^{j}\end{array}$ \\
\hline $3 \mathrm{~d} 4 \mathrm{p}^{1} \mathrm{~F}_{3}^{\mathrm{o}}$ & 32349.98 & 4.68 & & $\begin{array}{l}5.1 \pm 0.3^{d} \\
5.2 \pm 0.2^{e} \\
6.8 \pm 0.6^{h}\end{array}$ & $\begin{array}{l}4.46^{i} \\
5.20^{j}\end{array}$ \\
\hline $4 \mathrm{~s} 4 \mathrm{p}^{3} \mathrm{P}_{0}^{\mathrm{o}}$ & 39002.20 & 3.69 & & $3.7 \pm 0.2^{d}$ & $\begin{array}{l}3.36^{i} \\
3.66^{j}\end{array}$ \\
\hline $4 s 4 p^{3} P_{1}^{o}$ & 39115.04 & 3.69 & & $3.7 \pm 0.2^{d}$ & $\begin{array}{l}3.37^{i} \\
3.67^{j}\end{array}$ \\
\hline $4 s 4 p^{3} P_{2}^{o}$ & 39345.52 & 3.70 & & $3.8 \pm 0.2^{d}$ & $\begin{array}{l}3.39^{i} \\
3.67^{j}\end{array}$ \\
\hline $4 s 4 p{ }^{1} P_{1}^{o}$ & 55715.36 & 0.88 & & & $0.91^{i}$ \\
\hline $3 \mathrm{~d} 5 \mathrm{~s}^{3} \mathrm{D}_{1}$ & 57551.88 & 3.49 & & & $3.44^{i}$ \\
\hline $3 \mathrm{~d} 5 \mathrm{~s}^{3} \mathrm{D}_{2}$ & 57614.40 & 3.50 & & & $3.44^{i}$ \\
\hline $3 \mathrm{~d} 5 \mathrm{~s}^{3} \mathrm{D}_{3}$ & 57743.92 & 3.50 & $3.20 \pm 0.20$ & & $3.44^{i}$ \\
\hline $3 \mathrm{~d} 5 \mathrm{~s}^{1} \mathrm{D}_{2}$ & 58252.09 & 3.70 & $3.26 \pm 0.20$ & & $3.66^{j}$ \\
\hline $3 \mathrm{~d} 4 \mathrm{~d}^{1} \mathrm{~F}_{3}$ & 59528.42 & 2.69 & $2.32 \pm 0.15$ & & $\begin{array}{l}2.51^{i} \\
2.51^{j}\end{array}$ \\
\hline $3 \mathrm{~d} 4 \mathrm{~d}^{3} \mathrm{D}_{1}$ & 59875.08 & 2.72 & $2.23 \pm 0.15$ & & $2.62^{i}$ \\
\hline $3 \mathrm{~d} 4 \mathrm{~d}^{3} \mathrm{D}_{2}$ & 59929.46 & 2.74 & $2.32 \pm 0.15$ & & $\begin{array}{l}2.63^{i} \\
2.58^{j}\end{array}$ \\
\hline $3 \mathrm{~d} 4 \mathrm{~d}^{3} \mathrm{D}_{3}$ & 60001.91 & 2.76 & $2.41 \pm 0.20$ & & $2.65^{i}$ \\
\hline $3 \mathrm{~d} 4 \mathrm{~d}^{3} \mathrm{G}_{3}$ & 60267.16 & 2.50 & $2.19 \pm 0.15$ & $2.5 \pm 0.2^{d}$ & $\begin{array}{l}2.33^{i} \\
2.47^{j}\end{array}$ \\
\hline $3 \mathrm{~d} 4 \mathrm{~d}^{3} \mathrm{G}_{4}$ & 60348.46 & 2.52 & & $2.4 \pm 0.2^{d}$ & $\begin{array}{l}2.35^{i} \\
2.49^{j}\end{array}$ \\
\hline $3 \mathrm{~d} 4 \mathrm{~d}^{1} \mathrm{P}_{1}$ & 60400.41 & 2.89 & $2.44 \pm 0.15$ & & $\begin{array}{l}2.69^{i} \\
2.63^{j}\end{array}$ \\
\hline $3 \mathrm{~d} 4 \mathrm{~d}^{3} \mathrm{G}_{5}$ & 60457.12 & 2.54 & & $2.5 \pm 0.2^{d}$ & $\begin{array}{l}2.38^{i} \\
2.51^{j}\end{array}$ \\
\hline $3 \mathrm{~d} 4 \mathrm{~d}^{3} \mathrm{~S}_{1}$ & 61071.43 & 2.82 & $2.45 \pm 0.15$ & & $\begin{array}{l}2.77^{i} \\
2.78^{j}\end{array}$ \\
\hline $3 d 4 d^{3} F_{2}$ & 63374.63 & 2.40 & $2.15 \pm 0.10$ & & $\begin{array}{l}2.05^{i} \\
2.43^{j}\end{array}$ \\
\hline $3 \mathrm{~d} 4 \mathrm{~d}^{3} \mathrm{~F}_{3}$ & 63445.16 & 2.41 & & & $2.05^{i}$ \\
\hline $3 d 4 d^{3} F_{4}$ & 63528.54 & 2.43 & $2.19 \pm 0.10$ & & $\begin{array}{l}2.07^{i} \\
2.47^{j}\end{array}$ \\
\hline $3 \mathrm{~d} 4 \mathrm{~d}^{1} \mathrm{D}_{2}$ & 64366.68 & 2.73 & $2.25 \pm 0.15$ & & $2.26^{i}$ \\
\hline
\end{tabular}

Table 2 - continued

\begin{tabular}{lccccc}
\hline Level $^{a}$ & $\begin{array}{c}E^{a} \\
\left(\mathrm{~cm}^{-1}\right)\end{array}$ & $\begin{array}{c}\tau_{\text {this cal }}^{b} \\
(\mathrm{~ns})\end{array}$ & $\begin{array}{c}\tau_{\text {this exp }}^{c} \\
(\mathrm{~ns})\end{array}$ & $\begin{array}{c}\tau_{\text {other exp }} \\
(\mathrm{ns})\end{array}$ & $\begin{array}{c}\tau_{\text {other cal }} \\
(\mathrm{ns})\end{array}$ \\
\hline $3 \mathrm{~d} 4 \mathrm{~d}^{3} \mathrm{P}_{0}$ & 64615.77 & 3.21 & & $2.65^{i}$ \\
$3 \mathrm{~d} 4 \mathrm{~d}^{3} \mathrm{P}_{1}$ & 64646.70 & 3.21 & & $2.65^{i}$ \\
$3 \mathrm{~d} 4 \mathrm{~d}^{3} \mathrm{P}_{2}$ & 64705.89 & 3.19 & $2.51 \pm 0.15$ & $2.65^{i}$ \\
$3 \mathrm{~d} 4 \mathrm{~d}^{1} \mathrm{G}_{4}$ & 65236.04 & 3.17 & & $2.45^{i}$ \\
$3 \mathrm{~d} 4 \mathrm{~d}^{1} \mathrm{~S}_{0}$ & 67216.56 & 3.87 & & $2.74^{i}$ \\
$4 \mathrm{p}^{2}{ }^{1} \mathrm{D}_{2}$ & 74433.30 & 5.96 & $3.80 \pm 0.15$ & $6.80^{i}$ \\
$4 \mathrm{p}^{2}{ }^{3} \mathrm{P}_{0}$ & 76243.20 & 1.17 & & $1.28^{i}$ \\
$4 \mathrm{p}^{2}{ }^{3} \mathrm{P}_{1}$ & 76360.80 & 1.17 & $1.14 \pm 0.06$ & $1.28^{i}$ \\
$4 \mathrm{p}^{2}{ }^{3} \mathrm{P}_{2}$ & 76589.30 & 1.18 & $1.09 \pm 0.06$ & $1.30^{i}$ \\
& & & & $1.03^{j}$ \\
$3 \mathrm{~d} 6 \mathrm{~s}^{3} \mathrm{D}_{1}$ & 77195.19 & 5.56 & & $5.00^{i}$ \\
$3 \mathrm{~d} 6 \mathrm{~s}^{3} \mathrm{D}_{2}$ & 77256.99 & 5.55 & & $5.00^{i}$ \\
$3 \mathrm{~d} 6 \mathrm{~s}^{3} \mathrm{D}_{3}$ & 77387.17 & 5.54 & $3.73 \pm 0.25$ & & $4.98^{i}$ \\
$3 \mathrm{~d} 6 \mathrm{~s}{ }^{1} \mathrm{D}_{2}$ & 77833.88 & 6.61 & & $6.94^{i}$ \\
\hline
\end{tabular}

Notes. ${ }^{a}$ Johansson \& Litzén (1980).

${ }^{b} \mathrm{HFR}+\mathrm{CPOL}$ calculation, this work.

${ }^{c}$ TR-LIF measurements, this work.

${ }^{d}$ TR-LIF measurements by Marsden et al. (1988).

${ }^{e}$ TR-LIF measurements by Vogel et al. (1985).

${ }^{f}$ TR-LIF measurements by Arnesen et al. (1976).

${ }^{g}$ Beam-foil spectroscopy by Palenius et al. (1976).

${ }^{h}$ Beam-foil spectroscopy by Buchta et al. (1971).

${ }^{i}$ HFR calculation by Kurucz (2011).

${ }^{j}$ Parametric method calculation by Ruczkowski et al. (2014).

where $g_{\mathrm{u}}$ is the statistical weight of the upper level, $g_{1}$ the statistical weight of the lower level, $\lambda$ the wavelength of the transition in $\AA$ and $A_{\mathrm{ul}}$ the transition probability in $\mathrm{s}^{-1}$.

The transition probability is related to the branching fraction $(B F)$ and the lifetime of the upper level $\left(\tau_{\mathrm{u}}\right)$. It can be derived using

$A_{\mathrm{ul}}=\frac{B F_{\mathrm{ul}}}{\tau_{\mathrm{u}}}$.

We obtained the lifetimes of the upper levels from our measurements, as discussed in Section 2. The $B F$ is the parameter we measure and it is defined as the transition probability of the line, $A_{\mathrm{ul}}$, divided by the sum of transition probabilities of all lines from the same upper level;

$B F_{\mathrm{ul}}=\frac{A_{\mathrm{ul}}}{\sum_{i} A_{\mathrm{ui}}}=\frac{I_{\mathrm{ul}}}{\sum_{i} I_{\mathrm{ui}}}$.

Since all lines emanate from the same upper level, the transition probability is proportional to the line intensity, $I_{\mathrm{ul}}$, which for FTS spectra is proportional to photon flux (Davis, Abrams \& Brault 2001). Therefore, we derived $B F$ s from calibrated intensity ratios in our measurements. All lines were identified using the analysis of Johansson \& Litzén (1980). The intensities of the observed lines were determined by fitting Gaussian line profiles using GFIT (Engström 1998, 2014).

The uncertainty of the $A$-value, and thus of the $f$-value, arises from the uncertainty in the upper level lifetime and the uncertainty of the $B F$. The latter includes the uncertainty in the intensity calibration procedure and the uncertainty in the measured line intensity, including the self-absorption correction. The uncertainties of the integrated line intensities were determined using GFIT. The relative uncertainties are as low as 0.1 per cent for strong lines and 4 per cent on average. However, for two weak lines the uncertainty is as large as 20 percent. The uncertainty in the calibration using the tungsten lamp is 2.2 percent and the uncertainty using the deuterium lamp is 8.6 per cent for the region $425-210 \mathrm{~nm}$ and 9.9 per cent for 
Table 3. Presentation of experimental $\log (g f)$ values together with the transition, wavelength, $\lambda$, wavenumber, $\sigma$, measured branching fraction, $B F_{\text {exp }}$, experimental transition probability, $A_{\exp }$ and the corresponding rescaled semi-empirical $\log (g f)$ values of this work. The radiative lifetimes, $\tau$, are TR-LIF measurements from this work.

\begin{tabular}{|c|c|c|c|c|c|c|c|c|c|c|c|}
\hline \multicolumn{2}{|c|}{ Upper level $^{a}$} & \multicolumn{2}{|c|}{ Lower level $^{a}$} & \multirow{2}{*}{$\begin{array}{l}\lambda_{\exp }^{a} \\
(\mathrm{~nm})\end{array}$} & \multirow{2}{*}{$\begin{array}{c}\sigma_{\exp }^{a} \\
\left(\mathrm{~cm}^{-1}\right)\end{array}$} & \multirow{2}{*}{$\begin{array}{c}\sigma_{\text {theo }}^{b} \\
\left(\mathrm{~cm}^{-1}\right)\end{array}$} & \multirow[t]{2}{*}{$B F_{\exp }$} & \multirow{2}{*}{$\begin{array}{l}B F \text { unc. } \\
\text { per cent }\end{array}$} & \multirow{2}{*}{$\begin{array}{l}A_{\exp } \\
\left(\mathrm{s}^{-1}\right)\end{array}$} & \multirow{2}{*}{$\begin{array}{l}\log (g f) \\
\text { Exp. }\end{array}$} & \multirow{2}{*}{$\begin{array}{c}\log (g f)_{\text {resc }} \\
\text { Calc. }\end{array}$} \\
\hline Config. & Energy $\left(\mathrm{cm}^{-1}\right)$ & Config. & Energy $\left(\mathrm{cm}^{-1}\right)$ & & & & & & & & \\
\hline $\begin{array}{l}3 \mathrm{~d} 5 \mathrm{~s}^{3} \mathrm{D}_{3} \\
\tau=3.20 \pm 0.20 \mathrm{~ns}\end{array}$ & 57744 & $\begin{array}{l}3 \mathrm{~d} 4 \mathrm{p}^{3} \mathrm{~F}_{3}^{o} \\
3 \mathrm{~d} 4 \mathrm{p}^{3} \mathrm{~F}_{4}^{o} \\
3 \mathrm{~d} 4 \mathrm{p}^{3} \mathrm{D}_{2}^{o} \\
3 \mathrm{~d} 4 \mathrm{p}^{3} \mathrm{D}_{3}^{o} \\
3 \mathrm{~d} 4 \mathrm{p}^{3} \mathrm{P}_{2}^{o} \\
\text { Residual }\end{array}$ & $\begin{array}{l}27602 \\
27841 \\
28021 \\
28161 \\
29824\end{array}$ & $\begin{array}{l}331.673 \\
334.323 \\
336.347 \\
337.938 \\
358.064 \\
\end{array}$ & $\begin{array}{l}30141.50 \\
29902.57 \\
29722.58 \\
29582.76 \\
27919.88\end{array}$ & $\begin{array}{l}30176 \\
29944 \\
29771 \\
29620 \\
27888\end{array}$ & $\begin{array}{l}6.21 \mathrm{E}-02 \\
4.05 \mathrm{E}-01 \\
5.29 \mathrm{E}-02 \\
3.60 \mathrm{E}-01 \\
1.20 \mathrm{E}-01 \\
3.37 \mathrm{E}-03\end{array}$ & $\begin{array}{l}4 \\
3 \\
4 \\
3 \\
4\end{array}$ & $\begin{array}{l}1.94 \mathrm{E}+07 \\
1.27 \mathrm{E}+08 \\
1.65 \mathrm{E}+07 \\
1.12 \mathrm{E}+08 \\
3.75 \mathrm{E}+07\end{array}$ & $\begin{array}{r}-0.65 \pm 0.03 \\
0.17 \pm 0.03 \\
-0.71 \pm 0.03 \\
0.13 \pm 0.03 \\
-0.30 \pm 0.03\end{array}$ & $\begin{array}{r}-0.75 \\
0.14 \\
-0.69 \\
0.17 \\
-0.28\end{array}$ \\
\hline $\begin{array}{l}3 \mathrm{~d} 4 \mathrm{~d}^{1} \mathrm{~F}_{3} \\
\tau=2.32 \pm 0.15 \mathrm{~ns}\end{array}$ & 59528 & $\begin{array}{l}3 \mathrm{~d} 4 \mathrm{p}^{1} \mathrm{D}_{2}^{o} \\
3 \mathrm{~d} 4 \mathrm{p}^{3} \mathrm{D}_{3}^{o} \\
3 \mathrm{~d} 4 \mathrm{p}^{1} \mathrm{~F}_{3}^{o} \\
\text { Residual }\end{array}$ & $\begin{array}{l}26081 \\
28161 \\
32350\end{array}$ & $\begin{array}{l}298.893 \\
318.712 \\
367.834\end{array}$ & $\begin{array}{l}33447.17 \\
31367.21 \\
27178.50\end{array}$ & $\begin{array}{l}33296 \\
31408 \\
27121\end{array}$ & $\begin{array}{l}8.22 \mathrm{E}-01 \\
6.54 \mathrm{E}-03 \\
1.72 \mathrm{E}-01 \\
5.10 \mathrm{E}-03\end{array}$ & $\begin{array}{c}0.5 \\
14 \\
7\end{array}$ & $\begin{array}{l}3.54 \mathrm{E}+08 \\
2.82 \mathrm{E}+06 \\
7.40 \mathrm{E}+07\end{array}$ & $\begin{array}{r}0.52 \pm 0.03 \\
-1.52 \pm 0.06 \\
0.02 \pm 0.04\end{array}$ & $\begin{array}{r}0.47 \\
-1.45 \\
0.20\end{array}$ \\
\hline $\begin{array}{l}3 \mathrm{~d} 4 \mathrm{~d}^{3} \mathrm{D}_{2} \\
\tau=2.32 \pm 0.15 \mathrm{~ns}\end{array}$ & 59929 & $\begin{array}{l}3 \mathrm{~d} 4 \mathrm{p}^{3} \mathrm{~F}_{3}^{o} \\
3 \mathrm{~d} 4 \mathrm{p}^{3} \mathrm{D}_{1}^{o} \\
3 \mathrm{~d} 4 \mathrm{p}^{3} \mathrm{D}_{2}^{o} \\
3 \mathrm{~d} 4 \mathrm{p}^{3} \mathrm{D}_{3}^{o} \\
3 \mathrm{~d} 4 \mathrm{p}^{3} \mathrm{P}_{1}^{o} \\
3 \mathrm{~d} 4 \mathrm{p}^{3} \mathrm{P}_{2}^{o} \\
\text { Residual }\end{array}$ & $\begin{array}{l}27602 \\
27918 \\
28021 \\
28161 \\
29742 \\
29824\end{array}$ & $\begin{array}{l}309.249 \\
312.296 \\
313.309 \\
314.688 \\
331.170 \\
332.069\end{array}$ & $\begin{array}{l}32327.05 \\
32011.74 \\
31908.30 \\
31768.28 \\
30187.30 \\
30105.53\end{array}$ & $\begin{array}{l}32372 \\
32081 \\
31968 \\
31816 \\
30178 \\
30084\end{array}$ & $\begin{array}{l}1.21 \mathrm{E}-01 \\
8.39 \mathrm{E}-02 \\
4.12 \mathrm{E}-01 \\
6.61 \mathrm{E}-02 \\
2.50 \mathrm{E}-01 \\
6.67 \mathrm{E}-02 \\
3.42 \mathrm{E}-02\end{array}$ & $\begin{array}{l}5 \\
5 \\
3 \\
5 \\
4 \\
5\end{array}$ & $\begin{array}{l}5.23 \mathrm{E}+07 \\
3.62 \mathrm{E}+07 \\
1.78 \mathrm{E}+08 \\
2.85 \mathrm{E}+07 \\
1.08 \mathrm{E}+08 \\
2.87 \mathrm{E}+07\end{array}$ & $\begin{aligned}-0.43 & \pm 0.03 \\
-0.58 & \pm 0.03 \\
0.12 & \pm 0.03 \\
-0.67 & \pm 0.03 \\
-0.05 & \pm 0.03 \\
-0.62 & \pm 0.03\end{aligned}$ & $\begin{array}{r}-0.45 \\
-0.63 \\
0.06 \\
-0.68 \\
-0.02 \\
-0.56\end{array}$ \\
\hline $\begin{array}{l}3 \mathrm{~d} 4 \mathrm{~d}^{1} \mathrm{P}_{1} \\
\tau=2.44 \pm 0.15 \mathrm{~ns}\end{array}$ & 60400 & $\begin{array}{l}3 \mathrm{~d} 4 \mathrm{p}{ }^{1} \mathrm{D}_{2}^{o} \\
3 \mathrm{~d} 4 \mathrm{p}^{1} \mathrm{P}_{1}^{o} \\
\text { Residual }\end{array}$ & $\begin{array}{l}26081 \\
30816\end{array}$ & $\begin{array}{l}291.298 \\
337.915\end{array}$ & $\begin{array}{l}34319.09 \\
29584.65\end{array}$ & $\begin{array}{l}34206 \\
29673\end{array}$ & $\begin{array}{l}3.98 \mathrm{E}-01 \\
6.02 \mathrm{E}-01 \\
7.27 \mathrm{E}-02\end{array}$ & $\begin{array}{l}7 \\
5\end{array}$ & $\begin{array}{l}1.63 \mathrm{E}+08 \\
2.47 \mathrm{E}+08\end{array}$ & $\begin{array}{r}-0.21 \pm 0.04 \\
0.10 \pm 0.03\end{array}$ & $\begin{array}{r}-0.33 \\
0.14\end{array}$ \\
\hline $\begin{array}{l}3 \mathrm{~d} 4 \mathrm{~d}^{3} \mathrm{~S}_{1} \\
\tau=2.45 \pm 0.15 \mathrm{~ns}\end{array}$ & 61071 & $\begin{array}{l}3 \mathrm{~d} 4 \mathrm{p}^{3} \mathrm{P}_{0}^{o} \\
3 \mathrm{~d} 4 \mathrm{p}^{3} \mathrm{P}_{1}^{o} \\
3 \mathrm{~d} 4 \mathrm{p}^{3} \mathrm{P}_{2}^{o} \\
3 \mathrm{~d} 4 \mathrm{p}^{1} \mathrm{P}_{1}^{o} \\
\text { Residual }\end{array}$ & $\begin{array}{l}29736 \\
29742 \\
29824 \\
30816\end{array}$ & $\begin{array}{l}319.038 \\
319.098 \\
319.933 \\
330.421\end{array}$ & $\begin{array}{l}31335.12 \\
31329.24 \\
31247.50 \\
30255.76\end{array}$ & $\begin{array}{l}31336 \\
31326 \\
31231 \\
30319\end{array}$ & $\begin{array}{l}1.13 \mathrm{E}-01 \\
2.84 \mathrm{E}-01 \\
5.73 \mathrm{E}-01 \\
3.04 \mathrm{E}-02 \\
6.10 \mathrm{E}-02\end{array}$ & $\begin{array}{c}7 \\
6 \\
4 \\
10\end{array}$ & $\begin{array}{l}4.60 \mathrm{E}+07 \\
1.16 \mathrm{E}+08 \\
2.34 \mathrm{E}+08 \\
1.24 \mathrm{E}+07\end{array}$ & $\begin{aligned}-0.68 & \pm 0.04 \\
-0.28 & \pm 0.04 \\
0.03 & \pm 0.03 \\
-1.21 & \pm 0.05\end{aligned}$ & $\begin{array}{l}-0.70 \\
-0.25 \\
-0.02 \\
-1.28\end{array}$ \\
\hline $\begin{array}{l}3 \mathrm{~d} 4 \mathrm{~d}^{3} \mathrm{~F}_{2} \\
\tau=2.15 \pm 0.10 \mathrm{~ns}\end{array}$ & 63375 & $\begin{array}{c}3 \mathrm{~d} 4 \mathrm{p}^{3} \mathrm{~F}_{2}^{o} \\
3 \mathrm{~d} 4 \mathrm{p}^{3} \mathrm{~F}_{3}^{o} \\
3 \mathrm{~d} 4 \mathrm{p}^{3} \mathrm{D}_{1}^{o} \\
3 \mathrm{~d} 4 \mathrm{p}^{3} \mathrm{D}_{2}^{o} \\
\text { Residual }\end{array}$ & $\begin{array}{l}27444 \\
27602 \\
27918 \\
28021\end{array}$ & $\begin{array}{l}278.230 \\
279.464 \\
281.950 \\
282.776\end{array}$ & $\begin{array}{l}35930.81 \\
35772.19 \\
35456.96 \\
35353.30\end{array}$ & $\begin{array}{l}35960 \\
35802 \\
35511 \\
35398\end{array}$ & $\begin{array}{l}3.57 \mathrm{E}-01 \\
3.72 \mathrm{E}-02 \\
5.27 \mathrm{E}-01 \\
7.88 \mathrm{E}-02 \\
1.59 \mathrm{E}-02\end{array}$ & $\begin{array}{l}5 \\
9 \\
3 \\
6\end{array}$ & $\begin{array}{l}1.66 \mathrm{E}+08 \\
1.73 \mathrm{E}+07 \\
2.45 \mathrm{E}+08 \\
3.66 \mathrm{E}+07\end{array}$ & $\begin{aligned}-0.02 & \pm 0.03 \\
-0.99 & \pm 0.04 \\
0.17 & \pm 0.02 \\
-0.66 & \pm 0.03\end{aligned}$ & $\begin{array}{r}-0.07 \\
-0.95 \\
0.17 \\
-0.60\end{array}$ \\
\hline $\begin{array}{l}3 \mathrm{~d} 4 \mathrm{~d}^{3} \mathrm{~F}_{4} \\
\tau=2.19 \pm 0.10 \mathrm{~ns}\end{array}$ & 63529 & $\begin{array}{c}3 \mathrm{~d} 4 \mathrm{p}^{3} \mathrm{~F}_{4}^{o} \\
3 \mathrm{~d} 4 \mathrm{p}^{3} \mathrm{D}_{3}^{o} \\
\text { Residual }\end{array}$ & $\begin{array}{l}27841 \\
28161\end{array}$ & $\begin{array}{l}280.130 \\
282.663\end{array}$ & $\begin{array}{l}35687.12 \\
35367.30\end{array}$ & $\begin{array}{l}35726 \\
35402\end{array}$ & $\begin{array}{l}3.42 \mathrm{E}-01 \\
6.58 \mathrm{E}-01 \\
8.18 \mathrm{E}-03\end{array}$ & $\begin{array}{l}6 \\
3\end{array}$ & $\begin{array}{l}1.56 \mathrm{E}+08 \\
3.01 \mathrm{E}+08\end{array}$ & $\begin{array}{l}0.22 \pm 0.03 \\
0.51 \pm 0.02\end{array}$ & $\begin{array}{l}0.21 \\
0.51\end{array}$ \\
\hline $\begin{array}{l}3 \mathrm{~d} 4 \mathrm{~d}^{1} \mathrm{D}_{2} \\
\tau=2.25 \pm 0.15 \mathrm{~ns}\end{array}$ & 64367 & $\begin{array}{l}3 \mathrm{~d} 4 \mathrm{p}^{1} \mathrm{D}_{2}^{o} \\
3 \mathrm{~d} 4 \mathrm{p}^{3} \mathrm{~F}_{2}^{o} \\
3 \mathrm{~d} 4 \mathrm{p}^{3} \mathrm{P}_{1}^{o}\end{array}$ & $\begin{array}{l}26081 \\
27444 \\
29742\end{array}$ & $\begin{array}{l}261.119 \\
270.754 \\
288.728\end{array}$ & $\begin{array}{l}38285.22 \\
36923.00 \\
34624.48\end{array}$ & $\begin{array}{l}38187 \\
37012 \\
34661\end{array}$ & $\begin{array}{l}7.25 \mathrm{E}-01 \\
1.69 \mathrm{E}-02 \\
2.27 \mathrm{E}-02\end{array}$ & $\begin{array}{c}4 \\
12 \\
16\end{array}$ & $\begin{array}{l}3.22 \mathrm{E}+08 \\
7.51 \mathrm{E}+06 \\
1.01 \mathrm{E}+07\end{array}$ & $\begin{array}{r}0.22 \pm 0.03 \\
-1.38 \pm 0.06 \\
-1.20 \pm 0.07\end{array}$ & $\begin{array}{r}0.11 \\
-1.49 \\
-1.07\end{array}$ \\
\hline
\end{tabular}


Table 3 - continued

\begin{tabular}{|c|c|c|c|c|c|c|c|c|c|c|c|}
\hline \multicolumn{2}{|c|}{ Upper level $^{a}$} & \multicolumn{2}{|c|}{ Lower level $^{a}$} & \multirow{2}{*}{$\begin{array}{l}\lambda_{\exp }^{a} \\
(\mathrm{~nm})\end{array}$} & \multirow{2}{*}{$\begin{array}{c}\sigma_{\exp }^{a} \\
\left(\mathrm{~cm}^{-1}\right)\end{array}$} & \multirow{2}{*}{$\begin{array}{c}\sigma_{\text {theo }}^{b} \\
\left(\mathrm{~cm}^{-1}\right)\end{array}$} & \multirow[t]{2}{*}{$B F_{\exp }$} & \multirow{2}{*}{$\begin{array}{l}B F \text { unc. } \\
\text { per cent }\end{array}$} & \multirow{2}{*}{$\begin{array}{l}A_{\exp } \\
\left(\mathrm{s}^{-1}\right)\end{array}$} & \multirow{2}{*}{$\begin{array}{l}\log (g f) \\
\text { Exp. }\end{array}$} & \multirow{2}{*}{$\begin{array}{c}\log (g f)_{\text {resc }} \\
\text { Calc. }\end{array}$} \\
\hline Config. & Energy $\left(\mathrm{cm}^{-1}\right)$ & Config. & Energy $\left(\mathrm{cm}^{-1}\right)$ & & & & & & & & \\
\hline & & $\begin{array}{l}3 \mathrm{~d} 4 \mathrm{p}^{1} \mathrm{P}_{1}^{o} \\
\text { Residual }\end{array}$ & 30816 & 297.967 & 33550.90 & 33654 & $\begin{array}{l}2.35 \mathrm{E}-01 \\
4.94 \mathrm{E}-02\end{array}$ & 8 & $1.05 \mathrm{E}+08$ & $-0.16 \pm 0.04$ & -0.02 \\
\hline $\begin{array}{l}3 \mathrm{~d} 4 \mathrm{~d}^{3} \mathrm{P}_{2} \\
\tau=2.51 \pm 0.15 \mathrm{~ns}\end{array}$ & 64706 & $\begin{array}{l}3 \mathrm{~d} 4 \mathrm{p}^{3} \mathrm{D}_{3}^{o} \\
3 \mathrm{~d} 4 \mathrm{p}^{3} \mathrm{P}_{1}^{o} \\
3 \mathrm{~d} 4 \mathrm{p}^{3} \mathrm{P}_{2}^{o} \\
3 \mathrm{~d} 4 \mathrm{p}^{1} \mathrm{P}_{1}^{o} \\
\text { Residual }\end{array}$ & $\begin{array}{l}28161 \\
29742 \\
29824 \\
30816\end{array}$ & $\begin{array}{l}273.556 \\
285.927 \\
286.597 \\
294.984\end{array}$ & $\begin{array}{l}36544.66 \\
34963.68 \\
34881.86 \\
33890.19\end{array}$ & $\begin{array}{l}36597 \\
34960 \\
34865 \\
33953\end{array}$ & $\begin{array}{l}1.59 \mathrm{E}-01 \\
1.78 \mathrm{E}-01 \\
6.33 \mathrm{E}-01 \\
3.08 \mathrm{E}-02 \\
4.86 \mathrm{E}-02\end{array}$ & $\begin{array}{l}8 \\
6 \\
3 \\
8\end{array}$ & $\begin{array}{l}6.32 \mathrm{E}+07 \\
7.09 \mathrm{E}+07 \\
2.52 \mathrm{E}+08 \\
1.23 \mathrm{E}+07\end{array}$ & $\begin{array}{r}-0.45 \pm 0.04 \\
-0.36 \pm 0.04 \\
0.19 \pm 0.03 \\
-1.10 \pm 0.04\end{array}$ & $\begin{array}{r}-0.46 \\
-0.40 \\
0.16 \\
-0.98\end{array}$ \\
\hline $\begin{array}{l}4 \mathrm{p}^{2}{ }^{3} \mathrm{P}_{1} \\
\tau=1.14 \pm 0.06 \mathrm{~ns}\end{array}$ & 76361 & $\begin{array}{l}3 \mathrm{~d} 4 \mathrm{p}^{3} \mathrm{D}_{2}^{o} \\
4 \mathrm{~s} 4 \mathrm{p}^{3} \mathrm{P}_{0}^{o} \\
4 \mathrm{~s} 4 \mathrm{p}^{3} \mathrm{P}_{1}^{o} \\
4 \mathrm{~s} 4 \mathrm{p}^{3} \mathrm{P}_{2}^{o} \\
\text { Residual }\end{array}$ & $\begin{array}{l}28021 \\
39002 \\
39115 \\
39346\end{array}$ & $\begin{array}{l}206.804 \\
267.597 \\
268.407 \\
270.079\end{array}$ & $\begin{array}{l}48339.50 \\
37358.69 \\
37245.53 \\
37014.70\end{array}$ & $\begin{array}{l}48384 \\
37358 \\
37245 \\
37014\end{array}$ & $\begin{array}{l}3.16 \mathrm{E}-01 \\
2.29 \mathrm{E}-01 \\
1.80 \mathrm{E}-01 \\
2.75 \mathrm{E}-01 \\
6.46 \mathrm{E}-02\end{array}$ & $\begin{array}{l}7 \\
6 \\
6 \\
6\end{array}$ & $\begin{array}{l}2.77 \mathrm{E}+08 \\
2.01 \mathrm{E}+08 \\
1.58 \mathrm{E}+08 \\
2.41 \mathrm{E}+08\end{array}$ & $\begin{array}{l}-0.27 \pm 0.04 \\
-0.19 \pm 0.03 \\
-0.29 \pm 0.03 \\
-0.10 \pm 0.03\end{array}$ & $\begin{array}{l}-0.41 \\
-0.18 \\
-0.31 \\
-0.09\end{array}$ \\
\hline $\begin{array}{l}4 \mathrm{p}^{2}{ }^{3} \mathrm{P}_{2} \\
\tau=1.09 \pm 0.06 \mathrm{~ns}\end{array}$ & 76589 & $\begin{array}{l}3 \mathrm{~d} 4 \mathrm{p}^{3} \mathrm{D}_{2}^{o} \\
3 \mathrm{~d} 4 \mathrm{p}^{3} \mathrm{D}_{3}^{o} \\
4 \mathrm{~s} 4 \mathrm{p}^{3} \mathrm{P}_{1}^{o} \\
4 \mathrm{~s} 4 \mathrm{p}^{3} \mathrm{P}_{2}^{o} \\
\text { Residual }\end{array}$ & $\begin{array}{l}28021 \\
28161 \\
39115 \\
39346\end{array}$ & $\begin{array}{l}205.831 \\
206.426 \\
266.771 \\
268.422\end{array}$ & $\begin{array}{l}48568.03 \\
48428.15 \\
37474.35 \\
37243.72\end{array}$ & $\begin{array}{l}48615 \\
48464 \\
37477 \\
37246\end{array}$ & $\begin{array}{l}5.63 \mathrm{E}-02 \\
2.95 \mathrm{E}-01 \\
1.65 \mathrm{E}-01 \\
4.83 \mathrm{E}-01 \\
1.18 \mathrm{E}-02\end{array}$ & $\begin{array}{c}27 \\
6 \\
6 \\
4\end{array}$ & $\begin{array}{l}5.17 \mathrm{E}+07 \\
2.71 \mathrm{E}+08 \\
1.52 \mathrm{E}+08 \\
4.43 \mathrm{E}+08\end{array}$ & $\begin{aligned}-0.78 & \pm 0.11 \\
-0.06 & \pm 0.03 \\
-0.09 & \pm 0.03 \\
0.38 & \pm 0.03\end{aligned}$ & $\begin{array}{r}-0.88 \\
-0.13 \\
-0.06 \\
0.41\end{array}$ \\
\hline
\end{tabular}

Note. ${ }^{a}$ Energy level, wavelength and wavenumber values are taken from Johansson \& Litzén (1980) which are available in NIST data base (Kramida et al. 2015).

${ }^{b}$ Theoretical wavenumber values are from the calculations of this work.

317-158 $\mathrm{nm}$. These calibration lamp uncertainties include the calibration uncertainty and the variation resulting from the repeated measurements made before and after all scandium scans. The uncertainties of the radiative lifetimes are given in Table 2. Finally, we were not able to observe the weakest lines from the investigated level. However, we included their contributions as residuals with derived theoretical $B F$ s from our calculations. The residual $B F$ s are less than 7 percent for all levels. The uncertainties in the residuals were estimated to 50 per cent and included in the error budget. The final uncertainties in the oscillator strengths are presented in Table 4 and were derived from the individual contributions using the method described by Sikström et al. (2002).

\section{RADIATIVE PARAMETER CALCULATIONS}

To calculate branching fractions and the oscillator strengths in Sc II, we used the relativistic Hartree-Fock (HFR) method implemented in the Cowan's suite of atomic structure computer codes (Cowan 1981). It is modified by including a pseudo-potential and a correction to the electric dipole operator which take into account the core-polarization effects giving rise to the HFR+CPOL technique (Quinet et al. 1999).

In this study, the valence-valence correlation was included using the following configuration interaction (CI) expansions: $3 \mathrm{~d} 4 \mathrm{~s}+$ $3 d 5 s+3 d 6 s+3 d 7 s+3 d^{2}+3 d 4 d+3 d 5 d+3 d 6 d+3 d 7 d+3 d 5 g$ $+3 d 6 g+3 d 7 g+4 s^{2}+4 s 5 s+4 s 6 s+4 s 7 s+4 s 4 d+4 s 5 d+4 s 6 d$ $+4 s 7 d+4 s 5 g+4 s 6 g+4 s 7 g+4 p^{2}+4 d^{2}+4 f^{2}+4 p 4 f$ for the even parity and $3 d 4 p+3 d 5 p+3 d 6 p+3 d 7 p+3 d 4 f+3 d 5 f+$ $3 d 6 f+3 d 7 f+3 d 6 h+3 d 7 h+4 s 4 p+4 s 5 p+4 s 6 p+4 s 7 p+4 s 4 f$ $+4 s 5 f+4 s 6 f+4 s 7 f+4 s 6 h+4 s 7 h+4 p 4 d+4 d 4 f$ for the odd parity.

Regarding the core-polarization effects, a Sc IV $3 \mathrm{p}^{6}$ closedsubshell ionic core was considered where the dipole polarizability, $\alpha_{\mathrm{d}}=2.129 a_{0}^{3}$, was taken from the relativistic random-phase approximation calculations of Johnson, Kolb \& Huang (1983) and a cut-off radius of $1.17 a_{0}$ was estimated as the HFR mean radius of the outermost $3 p$ orbital, $\langle 3 p|r| 3 p\rangle_{\text {HFR }}$.
During a least-squares fit procedure, we adjusted some radial integrals to minimize the discrepancies between the Hamiltonian eigenvalues and the experimental energy levels taken from the NIST Atomic Spectra Database (Kramida et al. 2015). The latter are based on the term analysis originally carried out by Russell \& Meggers (1927) and later revised by Neufeld (1970) and by Johansson \& Litzén (1980). There are 168 levels belonging to the configurations $3 \mathrm{~d} 4 \mathrm{~s}, 3 \mathrm{~d}^{2}, 3 \mathrm{~d} 4 \mathrm{p}, 4 \mathrm{~s} 4 \mathrm{p}, 3 \mathrm{~d} 5 \mathrm{~s}, 3 \mathrm{~d} 4 \mathrm{~d}, 3 \mathrm{~d} 5 \mathrm{p}, 4 \mathrm{p}^{2}, 3 \mathrm{~d} 4 \mathrm{f}, 3 \mathrm{~d} 6 \mathrm{~s}, 4 \mathrm{~s} 5 \mathrm{~s}, 3 \mathrm{~d} 5 \mathrm{~d}$, $4 \mathrm{~s} 4 \mathrm{~d}, 3 \mathrm{~d} 5 \mathrm{f}, 3 \mathrm{~d} 5 \mathrm{~g}, 3 \mathrm{~d} 7 \mathrm{~s}, 3 \mathrm{~d} 6 \mathrm{~d}$ and $3 \mathrm{~d} 6 \mathrm{f}$. The average energies, $E_{\mathrm{av}}$, of the above-mentioned known configurations along with their direct, $F^{\mathrm{k}}$, exchange, $G^{\mathrm{k}}$, electrostatic and spin-orbit, $\zeta$, radial parameters were considered in the fit of the energy levels. The ab initio and fitted parameter values are reported in Tables 4 and 5 for the even and odd configurations, respectively. The spin-orbit integrals not presented in these tables were fixed to their HFR+CPOL values. The other Slater integrals, including the CI $R^{\mathrm{k}}$ parameters, not reported here, were fixed to 80 percent of their $a b$ initio values to account for missing CI effects (Cowan 1981). The average deviations of the least-squares fits were $157 \mathrm{~cm}^{-1}$ for the 93 even-parity experimental levels and $65 \mathrm{~cm}^{-1}$ for the 75 odd-parity experimental levels.

\section{RESULTS AND DISCUSSION}

Table 2 compares our TR-LIF and HFR+CPOL lifetimes with other experimental values from the literature (Buchta et al. 1971; Arnesen et al. 1976; Palenius et al. 1976; Vogel et al. 1985; Marsden et al. 1988), the HFR values calculated by Kurucz (2011) and the lifetimes deduced from the semi-empirical oscillator strengths calculated by Ruczkowski et al. (2014). On average, our HFR+CPOL lifetimes are shorter than the measurements for the odd-parity levels and longer for the even-parity levels. The discrepancies range from a few per cent to about 20 percent, except for the even-parity levels $4 \mathrm{p}^{2}{ }^{1} \mathrm{D}_{2}$ and $3 \mathrm{~d} 6 \mathrm{~s}^{3} \mathrm{D}_{3}$ where they reach 57 per cent and 49 per cent, respectively. In the former case, this state is strongly mixed (our calculation gives 36 per cent $4 \mathrm{p}^{2}{ }^{1} \mathrm{D}_{2}+36$ percent $4 \mathrm{~s} 4 \mathrm{~d}^{1} \mathrm{D}_{2}+23$ percent $\left.3 \mathrm{~d} 6 \mathrm{~s}^{1} \mathrm{D}_{2}\right)$ and an important decay channel $\left(4 \mathrm{p}^{2}{ }^{1} \mathrm{D}_{2} \rightarrow 3 \mathrm{~d} 4 \mathrm{p}^{1} \mathrm{D}_{2}^{\mathrm{o}} \quad B F=0.0713\right)$ is 
Table 4. Radial parameters adopted in the HFR + CPOL calculations for the even-parity configurations of $\mathrm{Sc}$ II. The parameters not listed here have been fixed to their ab initio values or to 80 per cent of their HFR+CPOL values for the electrostatic integrals.

\begin{tabular}{|c|c|c|c|c|c|}
\hline Config. & Parameter & $\begin{array}{c}\text { Ab initio } \\
\left(\mathrm{cm}^{-1}\right)\end{array}$ & $\begin{array}{l}\text { Fitted } \\
\left(\mathrm{cm}^{-1}\right)\end{array}$ & Ratio & Note $^{a}$ \\
\hline \multirow[t]{3}{*}{$3 \mathrm{~d} 4 \mathrm{~s}$} & $E_{\mathrm{av}}$ & 1075 & 1137 & & \\
\hline & $\zeta_{3 \mathrm{~d}}$ & 83 & 72 & 0.87 & \\
\hline & $G^{2}(3 \mathrm{~d} 4 \mathrm{~s})$ & 11351 & 9883 & 0.87 & \\
\hline \multirow[t]{3}{*}{$3 \mathrm{~d} 5 \mathrm{~s}$} & $E_{\mathrm{av}}$ & 57881 & 58144 & & \\
\hline & $\zeta_{3 \mathrm{~d}}$ & 87 & 79 & 0.91 & \\
\hline & $G^{2}(3 \mathrm{~d} 5 \mathrm{~s})$ & 2071 & 1851 & 0.89 & \\
\hline \multirow[t]{3}{*}{$3 \mathrm{~d} 6 \mathrm{~s}$} & $E_{\mathrm{av}}$ & 77397 & 77497 & & \\
\hline & $\zeta_{3 \mathrm{~d}}$ & 88 & 82 & 0.93 & \\
\hline & $G^{2}(3 \mathrm{~d} 6 \mathrm{~s})$ & 789 & 631 & 0.80 & $\mathrm{~F}$ \\
\hline \multirow[t]{3}{*}{$3 \mathrm{~d} 7 \mathrm{~s}$} & $E_{\mathrm{av}}$ & 86487 & 86549 & & \\
\hline & $\zeta_{3 \mathrm{~d}}$ & 88 & 69 & 0.78 & \\
\hline & $G^{2}(3 \mathrm{~d} 7 \mathrm{~s})$ & 393 & 314 & 0.80 & F \\
\hline \multirow[t]{7}{*}{$3 d^{2}$} & $E_{\mathrm{av}}$ & 11721 & 9531 & & \\
\hline & $F^{2}(3 \mathrm{~d} 3 \mathrm{~d})$ & 49657 & 37346 & 0.75 & \\
\hline & $F^{4}(3 \mathrm{~d} 3 \mathrm{~d})$ & 30556 & 22011 & 0.72 & \\
\hline & $\alpha$ & 0 & 64 & & \\
\hline & $\beta$ & 0 & -962 & & \\
\hline & $T$ & 0 & 3 & & \\
\hline & $\zeta_{3 \mathrm{~d}}$ & 65 & 59 & 0.91 & \\
\hline \multirow[t]{8}{*}{$3 \mathrm{~d} 4 \mathrm{~d}$} & $E_{\mathrm{av}}$ & 62210 & 62852 & & \\
\hline & $\zeta_{3 \mathrm{~d}}$ & 87 & 79 & 0.91 & \\
\hline & $\zeta_{4 \mathrm{~d}}$ & 8 & 8 & 1.00 & F \\
\hline & $F^{2}(3 \mathrm{~d} 4 \mathrm{~d})$ & 7539 & 5977 & 0.79 & \\
\hline & $F^{4}(3 \mathrm{~d} 4 \mathrm{~d})$ & 3599 & 2816 & 0.78 & \\
\hline & $G^{0}(3 \mathrm{~d} 4 \mathrm{~d})$ & 6862 & 2467 & 0.36 & \\
\hline & $G^{2}(3 \mathrm{~d} 4 \mathrm{~d})$ & 4352 & 3238 & 0.74 & \\
\hline & $G^{4}(3 \mathrm{~d} 4 \mathrm{~d})$ & 2927 & 2327 & 0.80 & \\
\hline \multirow[t]{8}{*}{$3 \mathrm{~d} 5 \mathrm{~d}$} & $E_{\mathrm{av}}$ & 79393 & 79170 & & \\
\hline & $\zeta_{3 \mathrm{~d}}$ & 87 & 86 & 0.99 & \\
\hline & $\zeta_{5 \mathrm{~d}}$ & 3 & 3 & 1.00 & F \\
\hline & $F^{2}(3 \mathrm{~d} 5 \mathrm{~d})$ & 2896 & 2158 & 0.75 & \\
\hline & $F^{4}(3 \mathrm{~d} 5 \mathrm{~d})$ & 1388 & 1099 & 0.79 & \\
\hline & $G^{0}(3 \mathrm{~d} 5 \mathrm{~d})$ & 2416 & 1008 & 0.42 & $\mathrm{R}$ \\
\hline & $G^{2}(3 \mathrm{~d} 5 \mathrm{~d})$ & 1640 & 684 & 0.42 & $\mathrm{R}$ \\
\hline & $G^{4}(3 \mathrm{~d} 5 \mathrm{~d})$ & 1122 & 469 & 0.42 & $\mathrm{R}$ \\
\hline \multirow[t]{8}{*}{$3 \mathrm{~d} 6 \mathrm{~d}$} & $E_{\mathrm{av}}$ & 87550 & 87894 & & \\
\hline & $\zeta_{3 \mathrm{~d}}$ & 88 & 88 & 1.00 & $\mathrm{~F}$ \\
\hline & $\zeta_{6 \mathrm{~d}}$ & 2 & 2 & 1.00 & $\mathrm{~F}$ \\
\hline & $F^{2}(3 \mathrm{~d} 6 \mathrm{~d})$ & 1458 & 1166 & 0.80 & $\mathrm{~F}$ \\
\hline & $F^{4}(3 \mathrm{~d} 6 \mathrm{~d})$ & 705 & 564 & 0.80 & $\mathrm{~F}$ \\
\hline & $G^{0}(3 \mathrm{~d} 6 \mathrm{~d})$ & 1176 & 941 & 0.80 & $\mathrm{~F}$ \\
\hline & $G^{2}(3 \mathrm{~d} 6 \mathrm{~d})$ & 822 & 658 & 0.80 & $\mathrm{~F}$ \\
\hline & $G^{4}(3 \mathrm{~d} 6 \mathrm{~d})$ & 571 & 454 & 0.80 & $\mathrm{~F}$ \\
\hline \multirow[t]{8}{*}{$3 \mathrm{~d} 5 \mathrm{~g}$} & $E_{\mathrm{av}}$ & 85492 & 85761 & & \\
\hline & $\zeta_{3 \mathrm{~d}}$ & 88 & 78 & 0.89 & \\
\hline & $\zeta_{5 \mathrm{~g}}$ & 0 & 0 & 1.00 & $\mathrm{~F}$ \\
\hline & $F^{2}(3 \mathrm{~d} 5 \mathrm{~g})$ & 465 & 420 & 0.90 & \\
\hline & $F^{4}(3 \mathrm{~d} 5 \mathrm{~g})$ & 42 & 34 & 0.81 & \\
\hline & $G^{2}(3 \mathrm{~d} 5 \mathrm{~g})$ & 6 & 5 & 0.80 & $\mathrm{~F}$ \\
\hline & $G^{4}(3 \mathrm{~d} 5 \mathrm{~g})$ & 4 & 3 & 0.80 & $\mathrm{~F}$ \\
\hline & $G^{6}(3 \mathrm{~d} 5 \mathrm{~g})$ & 2 & 2 & 0.80 & $\mathrm{~F}$ \\
\hline $4 s^{2}$ & $E_{\mathrm{av}}$ & 16845 & 16876 & & \\
\hline \multirow[t]{2}{*}{$4 \mathrm{~s} 5 \mathrm{~s}$} & $E_{\mathrm{av}}$ & 78974 & 79141 & & \\
\hline & $G^{0}(4 \mathrm{~s} 5 \mathrm{~s})$ & 2341 & 1765 & 0.75 & \\
\hline \multirow[t]{3}{*}{$4 s 4 d$} & $E_{\mathrm{av}}$ & 83034 & 82930 & & \\
\hline & $\zeta_{4 \mathrm{~d}}$ & 9 & 9 & 1.00 & F \\
\hline & $G^{2}(4 s 4 d)$ & 6830 & 5464 & 0.80 & $\mathrm{~F}$ \\
\hline \multirow[t]{3}{*}{$4 p^{2}$} & $E_{\mathrm{av}}$ & 77789 & 80625 & & \\
\hline & $F^{2}(4 \mathrm{p} 4 \mathrm{p})$ & 28516 & 29802 & 1.05 & \\
\hline & $\zeta_{4 p}$ & 199 & 253 & 1.27 & \\
\hline
\end{tabular}

Note. ${ }^{a} \mathrm{~F}$ and $\mathrm{R}$ stand for, respectively, a fixed parameter value and a fixed ratio between these parameters.
Table 5. Radial parameters adopted in the HFR + CPOL calculations for the odd-parity configurations of Sc II. The parameters not listed here have been fixed to their ab initio values or to 80 per cent of their HFR+CPOL values for the electrostatic integrals.

\begin{tabular}{|c|c|c|c|c|c|}
\hline Config. & Parameter & $\begin{array}{c}\text { Ab initio } \\
\left(\mathrm{cm}^{-1}\right)\end{array}$ & $\begin{array}{l}\text { Fitted } \\
\left(\mathrm{cm}^{-1}\right)\end{array}$ & Ratio & Note $^{a}$ \\
\hline \multirow[t]{6}{*}{$3 d 4 p$} & $E_{\mathrm{av}}$ & 28207 & 28996 & & \\
\hline & $\zeta_{3 \mathrm{~d}}$ & 85 & 91 & 1.07 & \\
\hline & $\zeta_{4 p}$ & 146 & 162 & 1.11 & \\
\hline & $F^{2}(3 \mathrm{~d} 4 \mathrm{p})$ & 14647 & 12024 & 0.82 & \\
\hline & $G^{1}(3 \mathrm{~d} 4 \mathrm{p})$ & 6709 & 6289 & 0.94 & \\
\hline & $G^{3}(3 \mathrm{~d} 4 \mathrm{p})$ & 5361 & 4338 & 0.81 & \\
\hline \multirow[t]{6}{*}{$3 d 5 p$} & $E_{\mathrm{av}}$ & 66759 & 66915 & & \\
\hline & $\zeta_{3 \mathrm{~d}}$ & 87 & 73 & 0.84 & \\
\hline & $\zeta_{5 p}$ & 50 & 50 & 1.00 & F \\
\hline & $F^{2}(3 \mathrm{~d} 5 \mathrm{p})$ & 4168 & 3375 & 0.81 & \\
\hline & $G^{1}(3 \mathrm{~d} 5 \mathrm{p})$ & 1560 & 1397 & 0.90 & \\
\hline & $G^{3}(3 \mathrm{~d} 5 \mathrm{p})$ & 1380 & 900 & 0.65 & \\
\hline \multirow[t]{8}{*}{$3 \mathrm{~d} 4 \mathrm{f}$} & $E_{\mathrm{av}}$ & 75021 & 75609 & & \\
\hline & $\zeta_{3 \mathrm{~d}}$ & 88 & 74 & 0.84 & \\
\hline & $\zeta_{4 \mathrm{f}}$ & 0 & 0 & 1.00 & F \\
\hline & $F^{2}(3 \mathrm{~d} 4 \mathrm{f})$ & 2127 & 1766 & 0.83 & \\
\hline & $F^{4}(3 \mathrm{~d} 4 \mathrm{f})$ & 514 & 367 & 0.71 & \\
\hline & $G^{1}(3 \mathrm{~d} 4 \mathrm{f})$ & 420 & 354 & 0.84 & \\
\hline & $G^{3}(3 \mathrm{~d} 4 \mathrm{f})$ & 242 & 194 & 0.80 & F \\
\hline & $G^{5}(3 \mathrm{~d} 4 \mathrm{f})$ & 166 & 133 & 0.80 & F \\
\hline \multirow[t]{8}{*}{$3 \mathrm{~d} 5 \mathrm{f}$} & $E_{\mathrm{av}}$ & 85220 & 85564 & & \\
\hline & $\zeta_{3 \mathrm{~d}}$ & 88 & 91 & 1.03 & \\
\hline & $\zeta_{5 f}$ & 0 & 0 & 1.00 & F \\
\hline & $F^{2}(3 \mathrm{~d} 5 \mathrm{f})$ & 1051 & 841 & 0.80 & F \\
\hline & $F^{4}(3 \mathrm{~d} 5 \mathrm{f})$ & 296 & 238 & 0.80 & F \\
\hline & $G^{1}(3 \mathrm{~d} 5 \mathrm{f})$ & 289 & 232 & 0.80 & $\mathrm{~F}$ \\
\hline & $G^{3}(3 \mathrm{~d} 5 \mathrm{f})$ & 168 & 135 & 0.80 & F \\
\hline & $G^{5}(3 \mathrm{~d} 5 \mathrm{f})$ & 116 & 93 & 0.80 & F \\
\hline \multirow[t]{8}{*}{$3 \mathrm{~d} 6 \mathrm{f}$} & $E_{\mathrm{av}}$ & 90728 & 91031 & & \\
\hline & $\zeta_{3 \mathrm{~d}}$ & 88 & 88 & 1.00 & F \\
\hline & $\zeta_{6 \mathrm{f}}$ & 0 & 0 & 1.00 & $\mathrm{~F}$ \\
\hline & $F^{2}(3 \mathrm{~d} 6 \mathrm{f})$ & 597 & 478 & 0.80 & F \\
\hline & $F^{4}(3 \mathrm{~d} 6 \mathrm{f})$ & 181 & 145 & 0.80 & $\mathrm{~F}$ \\
\hline & $G^{1}(3 \mathrm{~d} 6 \mathrm{f})$ & 188 & 151 & 0.80 & F \\
\hline & $G^{3}(3 \mathrm{~d} 6 \mathrm{f})$ & 111 & 88 & 0.80 & F \\
\hline & $G^{5}(3 \mathrm{~d} 6 \mathrm{f})$ & 76 & 61 & 0.80 & F \\
\hline \multirow[t]{3}{*}{$4 s 4 p$} & $E_{\mathrm{av}}$ & 41287 & 43719 & & \\
\hline & $\zeta_{4 \mathrm{p}}$ & 197 & 242 & 1.23 & \\
\hline & $G^{1}(4 s 4 p)$ & 37326 & 27686 & 0.74 & \\
\hline
\end{tabular}

Note. ${ }^{a} \mathrm{~F}$ stands for a fixed parameter value.

affected by cancellation (the cancellation factor as defined by Cowan (1981) is less than 5 per cent) that could explain the overestimated lifetime. Concerning $3 \mathrm{~d} 6 \mathrm{~s}^{3} \mathrm{D}_{3}$ level, no such argument could explain the observed disagreement. The beam-foil measurements of Buchta et al. (1971) can be rejected for the levels $3 \mathrm{~d} 4 \mathrm{p}{ }^{3} \mathrm{D}_{3}^{\mathrm{o}},{ }^{1} \mathrm{P}_{1}^{\mathrm{o} 1} \mathrm{~F}_{3}^{\mathrm{o}}$ as previously stated by Marsden et al. (1988) due to blending problems.

The calculations by Kurucz (2011) show roughly the same systematic discrepancy with experiment (lifetimes shorter for the odd parity and longer for the even parity) as our HFR+CPOL calculations. Although the calculation of Kurucz (2011) shows a better agreement than HFR+CPOL for certain $3 \mathrm{~d} 4 \mathrm{~d}$ levels $\left({ }^{3} \mathrm{~F}_{2,4},{ }^{1} \mathrm{D}_{2}\right.$ and ${ }^{3} \mathrm{P}_{2}$ ), it does not solve the theory-experiment disagreements observed for the levels $4 \mathrm{p}^{2}{ }^{1} \mathrm{D}_{2}$ and $3 \mathrm{~d} 6 \mathrm{~s}^{3} \mathrm{D}_{3}$. The parametric calculation of Ruczkowski et al. (2014) agrees with our HFR+CPOL model within 10 per cent including all levels. Unfortunately, no lifetime value can be deduced from Ruczkowski et al. (2014) for the levels $4 \mathrm{p}^{2}{ }^{1} \mathrm{D}_{2}$ and $3 \mathrm{~d} 6 \mathrm{~s}^{3} \mathrm{D}_{3}$. Concerning the level $3 \mathrm{~d} 4 \mathrm{~d}^{3} \mathrm{G}_{3}$, our 


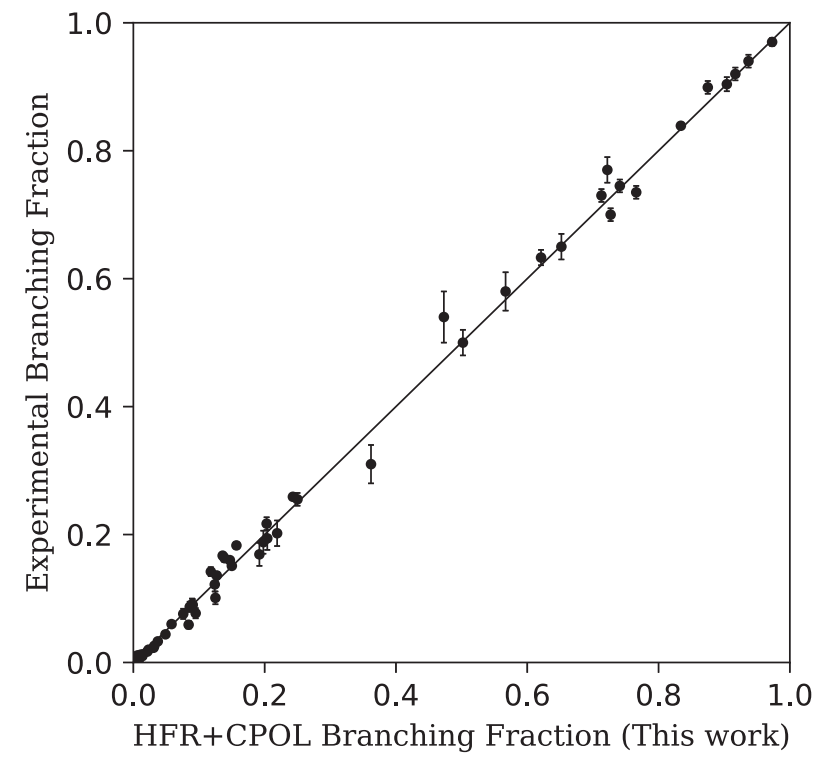

Figure 2. A comparison between the HFR+CPOL branching fractions of this work and the experimental values of Lawler \& Dakin (1989). The straight line of equality has been drawn.

TR-LIF measurement is slightly lower than the one of Marsden et al. (1988) although the error bars do overlap.

For all $3 \mathrm{~d} 4 \mathrm{p}$ levels, our HFR+CPOL model and the parametric calculation of Ruczkowski et al. (2014) are closer to the measurement of Marsden et al. (1988). The excellent agreement between Marsden et al. (1988) and Ruczkowski et al. (2014) is not surprising as the latter adjusted the dipole transition integrals to the oscillator strengths determined from the branching fraction measurements of Lawler \& Dakin (1989) combined with the lifetime measurements of Marsden et al. (1988). For most of the higher levels, the lifetimes calculated by Kurucz (2011) are closer to our measurements than those of Ruczkowski et al. (2014).

Although there is a systematic discrepancy between the theoretical and experimental lifetimes, we find a better agreement when comparing our calculated $B F \mathrm{~s}$ with the experimental values. For the high excitation lines, measured in this work, the averaged $B F$ ratio is $1.02 \pm 0.16$ with respect to the calculated values. Similarly, Fig. 2 shows the good agreement between $B F$ s computed in this study using the HFR+CPOL method and the measurements by Lawler \& Dakin (1989). Here, the averaged $B F$ ratio is $0.98 \pm 0.20$. Based on these comparisons, the calculated $B F$ s were combined with our TR-LIF lifetimes and those of Marsden et al. (1988) to determine rescaled transition probabilities and oscillator strengths.

In Table 3, we present our experimental $\log (g f)$ values, together with the measured $B F \mathrm{~s}$, the uncertainties and the corresponding rescaled theoretical oscillator strengths, $\log (g f)_{\text {resc }}$. Fig. 3 illustrates the final agreement between our experimental $\log (g f)$ values and the calculated $\log (g f)_{\text {resc }}$. Table 6 summarizes our calculated radiative parameters along with the weighted transition probabilities $(g A)$, the weighted oscillator strengths in the log scale $(\log (g f))$, the HFR+CPOL branching fractions $(B F)$, and the cancellation factor $(C F)$ as defined by Cowan (1981).

Our rescaled theoretical oscillator strengths are compared to the semi-empirical values calculated by Ruczkowski et al. (2014) in Fig. 4. As expected, the scatter increases for the weak lines, i.e. the transitions with $\log (g f) \lesssim-1$, where cancellation effects could be an issue. For instance, the transition $3 \mathrm{~d} 4 \mathrm{p}{ }^{3} \mathrm{P}_{2}^{\mathrm{o}}-4 \mathrm{p}^{2}{ }^{3} \mathrm{P}_{2}$ labelled in

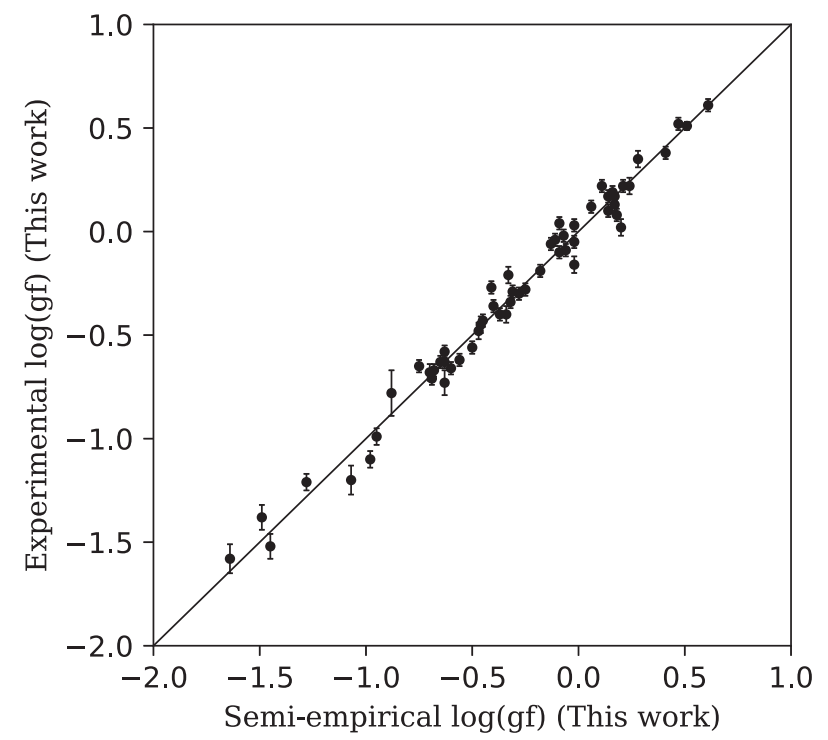

Figure 3. A comparison between the oscillator strengths determined by the combination of the HFR+CPOL branching fractions and the TR-LIF lifetimes of this work and the experimental oscillator strengths derived in this work. The straight line of equality has been drawn.

Table $676589(e) 2-29824(o) 2$ has a very low cancellation factor $(C F=0.001)$ that indicates a strong cancellation effect in our HFR+CPOL line strength calculation. Indeed, the rescaled oscillator strength for that transition is $\log (g f)_{\text {resc }}=-2.83$ which is three orders of magnitude lower (in the linear scale) than the value predicted by Ruczkowski et al. (2014) $(\log (g f)=-0.02)$. On the other hand, a transition for which the cancellation effect in our model is not an issue $(C F>0.05)$ such as $3 \mathrm{~d} 4 \mathrm{p}^{3} \mathrm{~F}_{3}^{\mathrm{o}}-3 \mathrm{~d} 4 \mathrm{~d}^{3} \mathrm{~F}_{4}$ $(63529(e) 4-27602(o) 3)$ has an oscillator strength predicted by Ruczkowski et al. (2014) $(\log (g f)=-3.35)$ that is two orders of magnitude lower than our rescaled value $\left(\log (g f)_{\text {resc }}=-1.44\right)$. This could indicate a strong cancellation effect in their calculation. Unfortunately, they did not estimate any cancellation factors. For the strongest transitions, i.e. $\log (g f) \gtrsim-1$, the mean scatter drops to about 20 per cent in the linear scale.

In Fig. 5, our semi-empirical values are compared to the calculation of Kurucz (2011) where a similar global correlation is observed. The mean scatter in this case is also found to be $\sim 20$ per cent for transitions with $\log (g f) \gtrsim-1$ and increases for weaker lines. Here again, the cancellation factors are not available in Kurucz's data base (Kurucz 2011). But, for example, our predicted strong line $3 \mathrm{~d} 5 \mathrm{p}^{3} \mathrm{~F}_{3}^{\mathrm{o}}-3 \mathrm{~d} 6 \mathrm{~s}^{3} \mathrm{D}_{3}(77387(e) 3-66564(o) 3)$ with $\log (g f)_{\text {resc }}=0.16$ and $C F=0.379$ is certainly affected by a strong cancellation effect in the calculation of Kurucz (2011) dramatically lowering its oscillator strength to $\log (g f)=-2.56$.

Based on the differences between different sets of $B F$ s discussed above and including the uncertainties of the experimental lifetimes, we estimate the accuracy of the rescaled theoretical $f$-values to be 10 per cent for the strong lines and 15-20 per cent for other lines.

\section{CONSEQUENCE ON THE SOLAR ABUNDANCE OF SCANDIUM}

Scott et al. (2015) have redetermined the solar abundances of the iron-peak elements employing a 3D model atmosphere that takes into account departures from the local thermodynamic equilibrium. However, the significant discrepancy between the photospheric and 
Table 6. Calculated branching fractions $(B F s)$, oscillator strengths $(\log (g f))$ and transition probabilities $(g A)$ along with the corresponding scaled values $\left(\log (g f)_{\text {resc }}, g A_{\text {resc }}\right)$ in $\mathrm{Sc}$ II. Only the transitions depopulating the levels for which the lifetime has been measured are listed. The experimental lifetimes $\left(\tau_{u}\right)$ used to scale the $f$ - and $A$-values are also reported.

\begin{tabular}{|c|c|c|c|c|c|c|c|c|c|c|c|c|c|}
\hline \multicolumn{3}{|c|}{ Upper level $^{a}$} & $\frac{\tau_{u}(\mathrm{~ns})}{7.5^{d}}$ & \multicolumn{3}{|c|}{ Lower level ${ }^{a}$} & $\begin{array}{l}\lambda^{b}(\mathrm{~nm}) \\
383.307\end{array}$ & $\begin{array}{c}B F \\
5.91 \mathrm{E}-03\end{array}$ & $\frac{g A\left(\mathrm{~s}^{-1}\right)}{4.44 \mathrm{E}+06}$ & $\frac{g A_{\text {resc }}\left(\mathrm{s}^{-1}\right)}{3.94 \mathrm{E}+06}$ & $\begin{array}{r}\log (g f) \\
-2.01\end{array}$ & $\frac{\log (g f)_{\text {resc }}}{-2.06}$ & $\frac{\mathrm{CF}^{c}}{0.468}$ \\
\hline & & & & 68 & (e) & 2 & 384.305 & $1.40 \mathrm{E}-02$ & $1.05 \mathrm{E}+07$ & $9.32 \mathrm{E}+06$ & -1.64 & -1.69 & 0.927 \\
\hline & & & & 2541 & (e) & 2 & 424.682 & $9.73 \mathrm{E}-01$ & $7.31 \mathrm{E}+08$ & $6.49 \mathrm{E}+08$ & 0.29 & 0.24 & 0.975 \\
\hline & & & & 4803 & (e) & 2 & 469.827 & $7.55 \mathrm{E}-04$ & $5.67 \mathrm{E}+05$ & $5.03 \mathrm{E}+05$ & -2.73 & -2.78 & 0.260 \\
\hline & & & & 4884 & (e) & 3 & 471.616 & $3.77 \mathrm{E}-05$ & $2.83 \mathrm{E}+04$ & $2.51 \mathrm{E}+04$ & -4.03 & -4.08 & 0.047 \\
\hline & & & & 10945 & (e) & 2 & 660.460 & $6.19 \mathrm{E}-03$ & $4.65 \mathrm{E}+06$ & $4.13 \mathrm{E}+06$ & -1.53 & -1.57 & 0.036 \\
\hline \multirow[t]{6}{*}{27444} & (o) & 2 & $6.2^{d}$ & 0 & (e) & 1 & 364.278 & $7.27 \mathrm{E}-01$ & $6.40 \mathrm{E}+08$ & $5.87 \mathrm{E}+08$ & 0.11 & 0.07 & 0.888 \\
\hline & & & & 68 & (e) & 2 & 365.180 & $1.57 \mathrm{E}-01$ & $1.38 \mathrm{E}+08$ & $1.26 \mathrm{E}+08$ & -0.56 & -0.60 & 0.894 \\
\hline & & & & 178 & (e) & 3 & 366.653 & $6.61 \mathrm{E}-03$ & $5.82 \mathrm{E}+06$ & $5.33 \mathrm{E}+06$ & -1.93 & -1.97 & 0.927 \\
\hline & & & & 2541 & (e) & 2 & 401.448 & $1.27 \mathrm{E}-02$ & $1.12 \mathrm{E}+07$ & $1.03 \mathrm{E}+07$ & -1.57 & -1.61 & 0.837 \\
\hline & & & & 4803 & (e) & 2 & 441.556 & $8.94 \mathrm{E}-02$ & $7.87 \mathrm{E}+07$ & $7.21 \mathrm{E}+07$ & -0.64 & -0.68 & 0.971 \\
\hline & & & & 4884 & (e) & 3 & 443.135 & $7.01 \mathrm{E}-03$ & $6.17 \mathrm{E}+06$ & $5.65 \mathrm{E}+06$ & -1.74 & -1.78 & 0.466 \\
\hline & & & & 178 & (e) & 3 & 364.531 & $1.36 \mathrm{E}-01$ & $1.69 \mathrm{E}+08$ & $1.56 \mathrm{E}+08$ & -0.47 & -0.51 & 0.952 \\
\hline & & & & 2541 & (e) & 2 & 398.906 & $7.51 \mathrm{E}-04$ & $9.35 \mathrm{E}+05$ & $8.62 \mathrm{E}+05$ & -2.65 & -2.69 & 0.768 \\
\hline & & & & 4803 & (e) & 2 & 438.481 & $7.95 \mathrm{E}-03$ & $9.90 \mathrm{E}+06$ & $9.12 \mathrm{E}+06$ & -1.54 & -1.58 & 0.966 \\
\hline & & & & 4884 & (e) & 3 & 440.039 & $8.59 \mathrm{E}-02$ & $1.07 \mathrm{E}+08$ & $9.86 \mathrm{E}+07$ & -0.51 & -0.54 & 0.975 \\
\hline & & & & 4988 & (e) & 4 & 442.067 & $3.37 \mathrm{E}-03$ & $4.20 \mathrm{E}+06$ & $3.87 \mathrm{E}+06$ & -1.91 & -1.95 & 0.242 \\
\hline & & & & 10945 & (e) & 2 & 600.150 & $4.67 \mathrm{E}-06$ & $5.82 \mathrm{E}+03$ & $5.36 \mathrm{E}+03$ & -4.50 & -4.54 & 0.520 \\
\hline & & & & 12154 & (e) & 2 & 647.153 & $4.47 \mathrm{E}-05$ & $5.57 \mathrm{E}+04$ & $5.13 \mathrm{E}+04$ & -3.46 & -3.49 & 0.612 \\
\hline & & & & 14261 & (e) & 4 & 749.355 & $5.08 \mathrm{E}-08$ & $6.33 \mathrm{E}+01$ & $5.83 \mathrm{E}+01$ & -6.28 & -6.31 & 0.089 \\
\hline 27841 & (o) & 4 & $6.1^{d}$ & 178 & (e) & 3 & 361.383 & $9.04 \mathrm{E}-01$ & $1.47 \mathrm{E}+09$ & $1.33 \mathrm{E}+09$ & 0.46 & 0.42 & 0.949 \\
\hline & & & & 4884 & (e) & 3 & 435.460 & $6.12 \mathrm{E}-03$ & $9.96 \mathrm{E}+06$ & $9.03 \mathrm{E}+06$ & -1.55 & -1.59 & 0.976 \\
\hline & & & & 4988 & (e) & 4 & 437.446 & $9.04 \mathrm{E}-02$ & $1.47 \mathrm{E}+08$ & $1.33 \mathrm{E}+08$ & -0.37 & -0.42 & 0.976 \\
\hline & & & & 14261 & (e) & 4 & 736.173 & $7.87 \mathrm{E}-07$ & $1.28 \mathrm{E}+03$ & $1.16 \mathrm{E}+03$ & -4.99 & -5.03 & 0.912 \\
\hline & & & & 12154 & (e) & 2 & 634.207 & $2.25 \mathrm{E}-04$ & $1.52 \mathrm{E}+05$ & $1.44 \mathrm{E}+05$ & -3.04 & -3.06 & 0.323 \\
\hline & & & & 25955 & (e) & 0 & 5093.945 & $6.48 \mathrm{E}-08$ & $4.38 \mathrm{E}+01$ & $4.14 \mathrm{E}+01$ & -4.72 & -4.79 & 0.157 \\
\hline 28021 & (o) & 2 & $4.7^{d}$ & 0 & (e) & 1 & 356.770 & $1.39 \mathrm{E}-01$ & $1.57 \mathrm{E}+08$ & $1.48 \mathrm{E}+08$ & -0.52 & -0.55 & 0.950 \\
\hline & & & & 68 & (e) & 2 & 357.634 & $5.02 \mathrm{E}-01$ & $5.68 \mathrm{E}+08$ & $5.34 \mathrm{E}+08$ & 0.04 & 0.01 & 0.857 \\
\hline & & & & 178 & (e) & 3 & 359.047 & $1.27 \mathrm{E}-01$ & $1.44 \mathrm{E}+08$ & $1.35 \mathrm{E}+08$ & -0.55 & -0.58 & 0.923 \\
\hline & & & & 2541 & (e) & 2 & 392.348 & $2.16 \mathrm{E}-03$ & $2.44 \mathrm{E}+06$ & $2.29 \mathrm{E}+06$ & -2.25 & -2.28 & 0.741 \\
\hline & & & & 4803 & (e) & 2 & 430.571 & $2.13 \mathrm{E}-02$ & $2.41 \mathrm{E}+07$ & $2.26 \mathrm{E}+07$ & -1.17 & -1.20 & 0.721 \\
\hline & & & & 4884 & (e) & 3 & 432.073 & $1.98 \mathrm{E}-01$ & $2.24 \mathrm{E}+08$ & $2.10 \mathrm{E}+08$ & -0.20 & -0.23 & 0.963 \\
\hline & & & & 10945 & (e) & 2 & 585.430 & $8.92 \mathrm{E}-07$ & $1.01 \mathrm{E}+03$ & $9.49 \mathrm{E}+02$ & -5.28 & -5.31 & 0.002 \\
\hline & & & & 12102 & (e) & 1 & 627.975 & $8.83 \mathrm{E}-03$ & $1.00 \mathrm{E}+07$ & $9.40 \mathrm{E}+06$ & -1.23 & -1.25 & 0.651 \\
\hline & & & & 12154 & (e) & 2 & 630.070 & $2.24 \mathrm{E}-03$ & $2.54 \mathrm{E}+06$ & $2.39 \mathrm{E}+06$ & -1.82 & -1.85 & 0.443 \\
\hline 28161 & (o) & 3 & $4.7^{d}$ & 68 & (e) & 2 & 355.853 & $1.18 \mathrm{E}-01$ & $1.88 \mathrm{E}+08$ & $1.76 \mathrm{E}+08$ & -0.45 & -0.48 & 0.968 \\
\hline & & & & 178 & (e) & 3 & 357.253 & $6.52 \mathrm{E}-01$ & $1.04 \mathrm{E}+09$ & $9.72 \mathrm{E}+08$ & 0.30 & 0.27 & 0.894 \\
\hline & & & & 2541 & (e) & 2 & 390.206 & $2.31 \mathrm{E}-05$ & $3.69 \mathrm{E}+04$ & $3.45 \mathrm{E}+04$ & -4.08 & -4.10 & 0.023 \\
\hline & & & & 4803 & (e) & 2 & 427.993 & $3.06 \mathrm{E}-04$ & $4.88 \mathrm{E}+05$ & $4.56 \mathrm{E}+05$ & -2.87 & -2.90 & 0.383 \\
\hline & & & & 4884 & (e) & 3 & 429.477 & $1.37 \mathrm{E}-02$ & $2.18 \mathrm{E}+07$ & $2.04 \mathrm{E}+07$ & -1.22 & -1.25 & 0.601 \\
\hline & & & & 4988 & (e) & 4 & 431.408 & $2.04 \mathrm{E}-01$ & $3.26 \mathrm{E}+08$ & $3.05 \mathrm{E}+08$ & -0.04 & -0.07 & 0.963 \\
\hline & & & & 10945 & (e) & 2 & 580.673 & $1.57 \mathrm{E}-04$ & $2.50 \mathrm{E}+05$ & $2.34 \mathrm{E}+05$ & -2.90 & -2.93 & 0.555 \\
\hline & & & & 12154 & (e) & 2 & 624.564 & $1.10 \mathrm{E}-02$ & $1.76 \mathrm{E}+07$ & $1.64 \mathrm{E}+07$ & -0.99 & -1.02 & 0.638 \\
\hline & & & & 14261 & (e) & 4 & 719.234 & $3.06 \mathrm{E}-05$ & $4.88 \mathrm{E}+04$ & $4.56 \mathrm{E}+04$ & -3.42 & -3.45 & 0.419 \\
\hline 29736 & (o) & 0 & $7.7^{d}$ & 0 & (e) & 1 & 336.193 & $8.75 \mathrm{E}-01$ & $1.38 \mathrm{E}+08$ & $1.14 \mathrm{E}+08$ & -0.63 & -0.72 & 0.519 \\
\hline & & & & 12102 & (e) & 1 & 566.904 & $1.25 \mathrm{E}-01$ & $1.98 \mathrm{E}+07$ & $1.63 \mathrm{E}+07$ & -1.02 & -1.10 & 0.885 \\
\hline
\end{tabular}


Table 6 - continued

\begin{tabular}{|c|c|c|c|c|c|c|c|c|c|c|c|c|c|}
\hline \multicolumn{3}{|c|}{ Upper level $^{a}$} & $\frac{\tau_{u}(\mathrm{~ns})}{7.6^{d}}$ & \multicolumn{3}{|c|}{ Lower level ${ }^{a}$} & $\begin{array}{l}\lambda^{b}(\mathrm{~nm}) \\
336.127\end{array}$ & $\frac{B F}{2.43 \mathrm{E}-01}$ & $\frac{g A\left(\mathrm{~s}^{-1}\right)}{1.14 \mathrm{E}+08}$ & $\frac{g A_{\text {resc }}\left(\mathrm{s}^{-1}\right)}{9.57 \mathrm{E}+07}$ & $\begin{array}{r}\log (g f) \\
-0.72\end{array}$ & $\begin{array}{c}\log (g f)_{\text {resc }} \\
-0.79\end{array}$ & $\frac{\mathrm{CF}^{c}}{0.538}$ \\
\hline & & & & 68 & (e) & 2 & 336.894 & $6.21 \mathrm{E}-01$ & $2.92 \mathrm{E}+08$ & $2.45 \mathrm{E}+08$ & -0.30 & -0.38 & 0.485 \\
\hline & & & & 4803 & (e) & 2 & 400.860 & $2.21 \mathrm{E}-04$ & $1.04 \mathrm{E}+05$ & $8.73 \mathrm{E}+04$ & -3.60 & -3.68 & 0.479 \\
\hline & & & & 10945 & (e) & 2 & 531.835 & $8.60 \mathrm{E}-03$ & $4.04 \mathrm{E}+06$ & $3.39 \mathrm{E}+06$ & -1.77 & -1.84 & 0.723 \\
\hline & & & & 11736 & (e) & 0 & 555.222 & $5.30 \mathrm{E}-03$ & $2.49 \mathrm{E}+06$ & $2.09 \mathrm{E}+06$ & -1.94 & -2.01 & 0.771 \\
\hline & & & & 12074 & (e) & 0 & 565.836 & $3.72 \mathrm{E}-02$ & $1.75 \mathrm{E}+07$ & $1.47 \mathrm{E}+07$ & -1.08 & -1.15 & 0.736 \\
\hline & & & & 12154 & (e) & 2 & 568.420 & $4.89 \mathrm{E}-02$ & $2.30 \mathrm{E}+07$ & $1.93 \mathrm{E}+07$ & -0.96 & -1.03 & 0.813 \\
\hline & & & & 25955 & (e) & 0 & 2639.920 & $7.02 \mathrm{E}-06$ & $3.30 \mathrm{E}+03$ & $2.77 \mathrm{E}+03$ & -3.46 & -3.54 & 0.175 \\
\hline 29824 & (o) & 2 & $7.4^{d}$ & 0 & (e) & 1 & 335.205 & $1.05 \mathrm{E}-02$ & $8.36 \mathrm{E}+06$ & $7.12 \mathrm{E}+06$ & -1.85 & -1.92 & 0.529 \\
\hline & & & & 68 & (e) & 2 & 335.968 & $1.47 \mathrm{E}-01$ & $1.17 \mathrm{E}+08$ & $9.96 \mathrm{E}+07$ & -0.71 & -0.77 & 0.537 \\
\hline & & & & 178 & (e) & 3 & 337.215 & $7.13 \mathrm{E}-01$ & $5.66 \mathrm{E}+08$ & $4.82 \mathrm{E}+08$ & -0.02 & -0.09 & 0.506 \\
\hline & & & & 2541 & (e) & 2 & 366.425 & $2.42 \mathrm{E}-03$ & $1.92 \mathrm{E}+06$ & $1.63 \mathrm{E}+06$ & -2.42 & -2.48 & 0.731 \\
\hline & & & & 4803 & (e) & 2 & 399.550 & $3.24 \mathrm{E}-05$ & $2.57 \mathrm{E}+04$ & $2.19 \mathrm{E}+04$ & -4.21 & -4.28 & 0.865 \\
\hline 30816 & (o) & 1 & $8.8^{d}$ & 0 & (e) & 1 & 324.416 & $7.98 \mathrm{E}-04$ & $2.95 \mathrm{E}+05$ & $2.72 \mathrm{E}+05$ & -3.33 & -3.37 & 0.025 \\
\hline & & & & 68 & (e) & 2 & 325.131 & $2.31 \mathrm{E}-02$ & $8.56 \mathrm{E}+06$ & $7.89 \mathrm{E}+06$ & -1.87 & -1.90 & 0.259 \\
\hline & & & & 2541 & (e) & 2 & 353.571 & $4.73 \mathrm{E}-01$ & $1.75 \mathrm{E}+08$ & $1.61 \mathrm{E}+08$ & -0.49 & -0.52 & 0.193 \\
\hline & & & & 4803 & (e) & 2 & 384.317 & $2.68 \mathrm{E}-04$ & $9.91 \mathrm{E}+04$ & $9.14 \mathrm{E}+04$ & -3.66 & -3.69 & 0.065 \\
\hline & & & & 10945 & (e) & 2 & 503.102 & $3.62 \mathrm{E}-01$ & $1.34 \mathrm{E}+08$ & $1.24 \mathrm{E}+08$ & -0.29 & -0.33 & 0.723 \\
\hline & & & & 11736 & (e) & 0 & 523.981 & $1.24 \mathrm{E}-01$ & $4.60 \mathrm{E}+07$ & $4.24 \mathrm{E}+07$ & -0.72 & -0.76 & 0.685 \\
\hline & & & & 12074 & (e) & 0 & 533.424 & $8.14 \mathrm{E}-03$ & $3.01 \mathrm{E}+06$ & $2.77 \mathrm{E}+06$ & -1.89 & -1.93 & 0.773 \\
\hline & & & & 12102 & (e) & 1 & 534.205 & $7.73 \mathrm{E}-04$ & $2.86 \mathrm{E}+05$ & $2.64 \mathrm{E}+05$ & -2.91 & -2.95 & 0.422 \\
\hline & & & & 12154 & (e) & 2 & 535.720 & $6.06 \mathrm{E}-03$ & $2.24 \mathrm{E}+06$ & $2.06 \mathrm{E}+06$ & -2.01 & -2.05 & 0.758 \\
\hline & & & & 25955 & (e) & 0 & 2056.840 & $8.71 \mathrm{E}-04$ & $3.22 \mathrm{E}+05$ & $2.97 \mathrm{E}+05$ & -1.67 & -1.73 & 0.214 \\
\hline 32350 & (o) & 3 & $5.1^{d}$ & 68 & (e) & 2 & 309.678 & $5.40 \mathrm{E}-04$ & $8.08 \mathrm{E}+05$ & $7.41 \mathrm{E}+05$ & -2.94 & -2.97 & 0.182 \\
\hline & & & & 178 & (e) & 3 & 310.737 & $5.99 \mathrm{E}-04$ & $8.97 \mathrm{E}+05$ & $8.22 \mathrm{E}+05$ & -2.89 & -2.92 & 0.835 \\
\hline & & & & 12102 & (e) & 1 & 371.632 & $5.85 \mathrm{E}-03$ & $1.59 \mathrm{E}+06$ & $1.58 \mathrm{E}+06$ & -2.48 & -2.48 & 0.139 \\
\hline 39115 & (o) & 1 & $3.7^{d}$ & 0 & (e) & 1 & 255.580 & $2.50 \mathrm{E}-01$ & $2.04 \mathrm{E}+08$ & $2.03 \mathrm{E}+08$ & -0.70 & -0.70 & 0.958 \\
\hline & & & & 68 & (e) & 2 & 256.023 & $7.41 \mathrm{E}-01$ & $6.04 \mathrm{E}+08$ & $6.01 \mathrm{E}+08$ & -0.23 & -0.23 & 0.955 \\
\hline & & & & 2541 & (e) & 2 & 273.337 & $4.16 \mathrm{E}-04$ & $3.39 \mathrm{E}+05$ & $3.37 \mathrm{E}+05$ & -3.42 & -3.42 & 0.244 \\
\hline & & & & 4803 & (e) & 2 & 291.357 & $4.23 \mathrm{E}-07$ & $3.45 \mathrm{E}+02$ & $3.43 \mathrm{E}+02$ & -6.36 & -6.36 & 0.362 \\
\hline & & & & 10945 & (e) & 2 & 354.880 & $2.80 \mathrm{E}-05$ & $2.28 \mathrm{E}+04$ & $2.27 \mathrm{E}+04$ & -4.37 & -4.37 & 0.095 \\
\hline & & & & 11736 & (e) & 0 & 365.144 & $5.13 \mathrm{E}-07$ & $4.18 \mathrm{E}+02$ & $4.16 \mathrm{E}+02$ & -6.08 & -6.08 & 0.001 \\
\hline & & & & 12074 & (e) & 0 & 369.704 & $1.94 \mathrm{E}-03$ & $1.58 \mathrm{E}+06$ & $1.57 \mathrm{E}+06$ & -2.49 & -2.49 & 0.137 \\
\hline & & & & 12102 & (e) & 1 & 370.079 & $1.44 \mathrm{E}-03$ & $1.17 \mathrm{E}+06$ & $1.16 \mathrm{E}+06$ & -2.62 & -2.62 & 0.137 \\
\hline & & & & 12154 & (e) & 2 & 370.806 & $2.36 \mathrm{E}-03$ & $1.92 \mathrm{E}+06$ & $1.91 \mathrm{E}+06$ & -2.40 & -2.40 & 0.136 \\
\hline & & & & 25955 & (e) & 0 & 759.679 & $5.27 \mathrm{E}-07$ & $4.29 \mathrm{E}+02$ & $4.27 \mathrm{E}+02$ & -5.43 & -5.43 & 0.074 \\
\hline 39346 & (o) & 2 & $3.8^{d}$ & 0 & (e) & 1 & 254.082 & $1.02 \mathrm{E}-02$ & $1.38 \mathrm{E}+07$ & $1.34 \mathrm{E}+07$ & -1.88 & -1.89 & 0.957 \\
\hline & & & & 68 & (e) & 2 & 254.520 & $1.50 \mathrm{E}-01$ & $2.04 \mathrm{E}+08$ & $1.98 \mathrm{E}+08$ & -0.70 & -0.72 & 0.957 \\
\hline & & & & 178 & (e) & 3 & 255.235 & $8.34 \mathrm{E}-01$ & $1.13 \mathrm{E}+09$ & $1.10 \mathrm{E}+09$ & 0.04 & 0.03 & 0.956 \\
\hline & & & & 2541 & (e) & 2 & 271.625 & $2.29 \mathrm{E}-04$ & $3.10 \mathrm{E}+05$ & $3.01 \mathrm{E}+05$ & -3.47 & -3.48 & 0.829 \\
\hline & & & & 4803 & (e) & 2 & 289.412 & $1.49 \mathrm{E}-07$ & $2.02 \mathrm{E}+02$ & $1.96 \mathrm{E}+02$ & -6.60 & -6.61 & 0.745 \\
\hline & & & & 4884 & (e) & 3 & 290.090 & $4.46 \mathrm{E}-07$ & $6.05 \mathrm{E}+02$ & $5.87 \mathrm{E}+02$ & -6.12 & -6.13 & 0.917 \\
\hline & & & & 10945 & (e) & 2 & 352.000 & $2.32 \mathrm{E}-05$ & $3.14 \mathrm{E}+04$ & $3.05 \mathrm{E}+04$ & -4.23 & -4.25 & 0.097 \\
\hline & & & & 12102 & (e) & 1 & 366.949 & $1.36 \mathrm{E}-03$ & $1.85 \mathrm{E}+06$ & $1.80 \mathrm{E}+06$ & -2.43 & -2.44 & 0.129 \\
\hline & & & & 12154 & (e) & 2 & 367.663 & $4.12 \mathrm{E}-03$ & $5.58 \mathrm{E}+06$ & $5.42 \mathrm{E}+06$ & -1.95 & -1.96 & 0.132 \\
\hline 57744 & (e) & 3 & $3.20^{e}$ & 26081 & (o) & 2 & 315.739 & $5.95 \mathrm{E}-06$ & $1.19 \mathrm{E}+04$ & $1.30 \mathrm{E}+04$ & -4.75 & -4.71 & 0.004 \\
\hline & & & & 27444 & (o) & 2 & 329.936 & $1.75 \mathrm{E}-03$ & $3.49 \mathrm{E}+06$ & $3.82 \mathrm{E}+06$ & -2.25 & -2.21 & 0.660 \\
\hline & & & & 27602 & (o) & 3 & 331.673 & $4.87 \mathrm{E}-02$ & $9.74 \mathrm{E}+07$ & $1.07 \mathrm{E}+08$ & -0.80 & -0.75 & 0.694 \\
\hline & & & & 27841 & (o) & 4 & 334.323 & $3.77 \mathrm{E}-01$ & $7.53 \mathrm{E}+08$ & $8.24 \mathrm{E}+08$ & 0.10 & 0.14 & 0.668 \\
\hline
\end{tabular}


Table 6 - continued

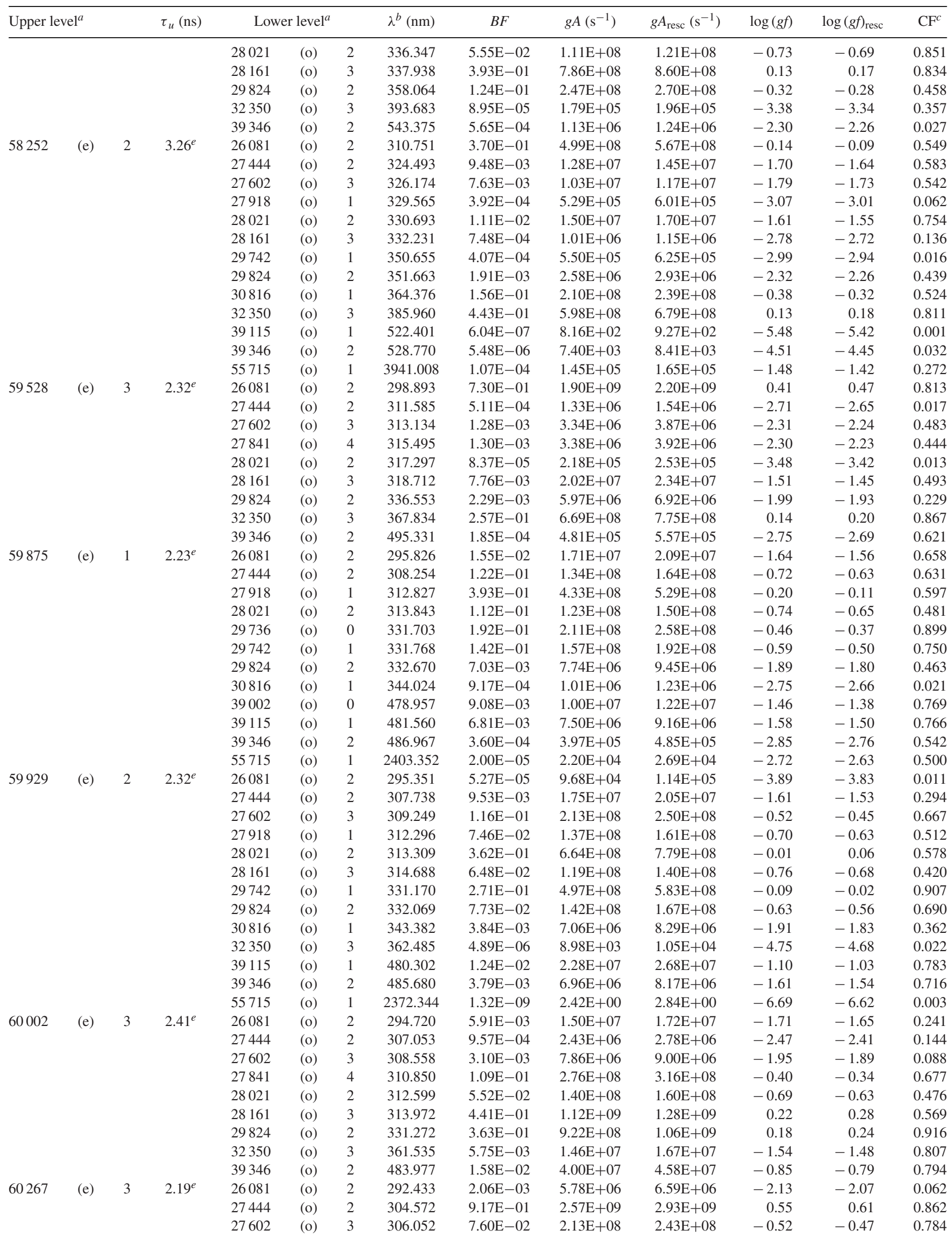


Table 6 - continued

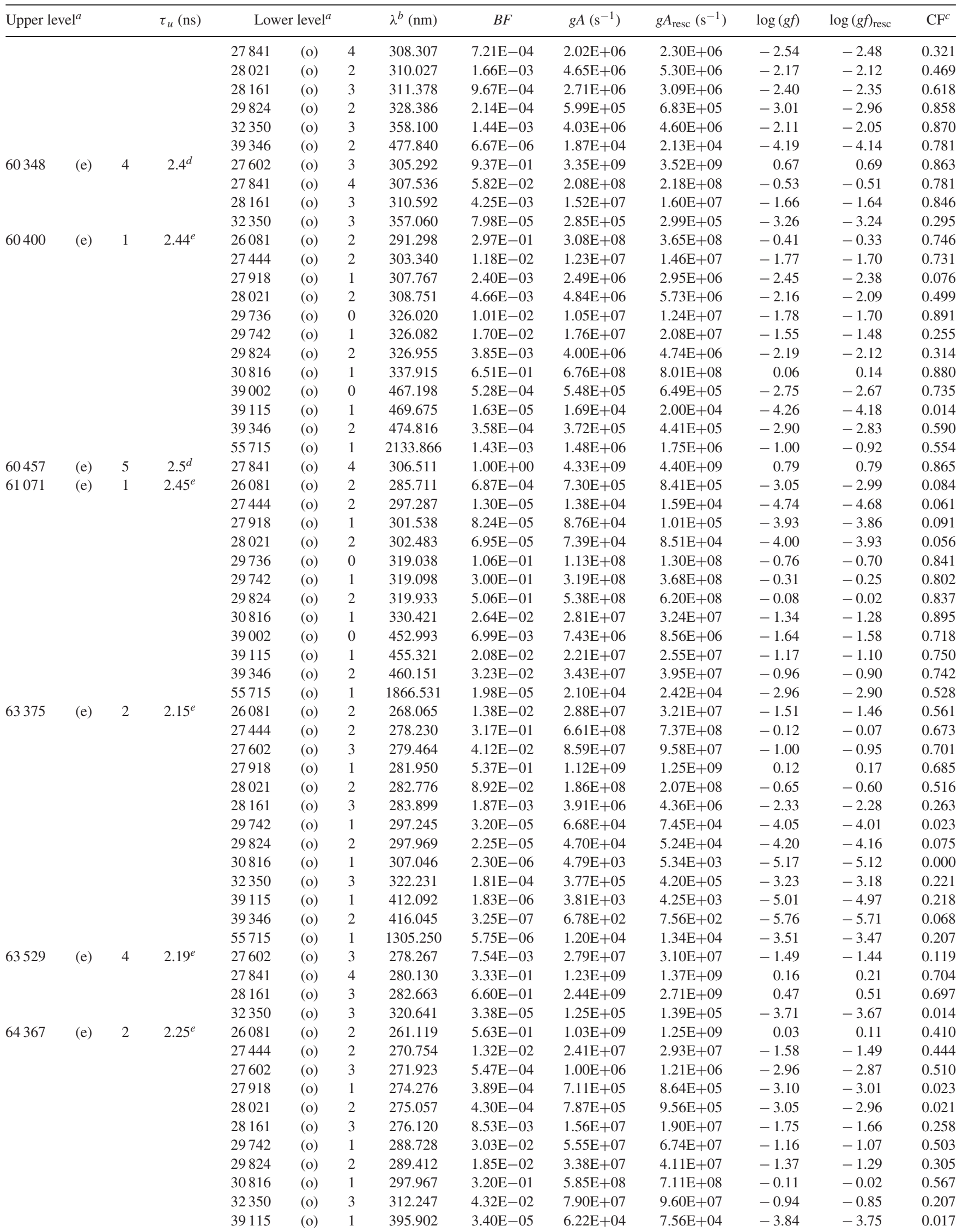


Table 6 - continued

\begin{tabular}{|c|c|c|c|c|c|c|c|c|c|c|c|c|c|}
\hline \multicolumn{3}{|c|}{ Upper level $^{a}$} & \multirow{2}{*}{$\begin{array}{c}\tau_{u}(\mathrm{~ns}) \\
2.51^{e}\end{array}$} & \multicolumn{3}{|c|}{ Lower level $^{a}$} & $\frac{\lambda^{b}(\mathrm{~nm})}{399.549}$ & $\frac{B F}{9.57 \mathrm{E}-05}$ & $\frac{g A\left(\mathrm{~s}^{-1}\right)}{1.75 \mathrm{E}+05}$ & $\frac{g A_{\text {resc }}\left(\mathrm{s}^{-1}\right)}{2.13 \mathrm{E}+05}$ & $\begin{array}{r}\log (g f) \\
-3.38\end{array}$ & $\begin{array}{c}\log (g f)_{\text {resc }} \\
-3.29\end{array}$ & $\frac{\mathrm{CF}^{c}}{0.021}$ \\
\hline & & & & 55715 & (o) & 1 & 1155.577 & $1.90 \mathrm{E}-03$ & $3.48 \mathrm{E}+06$ & $4.23 \mathrm{E}+06$ & -1.16 & -1.07 & 0.207 \\
\hline & & & & 27444 & (o) & 2 & 268.289 & $8.23 \mathrm{E}-04$ & $1.29 \mathrm{E}+06$ & $1.64 \mathrm{E}+06$ & -2.86 & -2.75 & 0.238 \\
\hline & & & & 27602 & (o) & 3 & 269.437 & $6.38 \mathrm{E}-04$ & $9.99 \mathrm{E}+05$ & $1.27 \mathrm{E}+06$ & -2.97 & -2.86 & 0.208 \\
\hline & & & & 27918 & (o) & 1 & 271.746 & $8.55 \mathrm{E}-04$ & $1.34 \mathrm{E}+06$ & $1.70 \mathrm{E}+06$ & -2.83 & -2.72 & 0.055 \\
\hline & & & & 28021 & (o) & 2 & 272.513 & $2.21 \mathrm{E}-02$ & $3.46 \mathrm{E}+07$ & $4.40 \mathrm{E}+07$ & -1.42 & -1.31 & 0.154 \\
\hline & & & & 29742 & (o) & 1 & 285.927 & $1.63 \mathrm{E}-01$ & $2.56 \mathrm{E}+08$ & $3.26 \mathrm{E}+08$ & -0.50 & -0.40 & 0.424 \\
\hline & & & & 29824 & (o) & 2 & 286.597 & $5.87 \mathrm{E}-01$ & $9.20 \mathrm{E}+08$ & $1.17 \mathrm{E}+09$ & 0.06 & 0.16 & 0.545 \\
\hline & & & & 30816 & (o) & 1 & 294.984 & $4.05 \mathrm{E}-02$ & $6.35 \mathrm{E}+07$ & $8.07 \mathrm{E}+07$ & -1.08 & -0.98 & 0.562 \\
\hline & & & & 32350 & (o) & 3 & 308.973 & $3.45 \mathrm{E}-03$ & $5.40 \mathrm{E}+06$ & $6.87 \mathrm{E}+06$ & -2.11 & -2.01 & 0.222 \\
\hline & & & & 39115 & (o) & 1 & 390.654 & $7.53 \mathrm{E}-04$ & $1.18 \mathrm{E}+06$ & $1.50 \mathrm{E}+06$ & -2.57 & -2.46 & 0.021 \\
\hline & & & & 39346 & (o) & 2 & 394.204 & $2.45 \mathrm{E}-03$ & $3.84 \mathrm{E}+06$ & $4.88 \mathrm{E}+06$ & -2.05 & -1.94 & 0.024 \\
\hline & & & & 55715 & (o) & 1 & 1111.977 & $1.19 \mathrm{E}-04$ & $1.87 \mathrm{E}+05$ & $2.38 \mathrm{E}+05$ & -2.46 & -2.36 & 0.198 \\
\hline & & & & 28021 & (o) & 2 & 215.394 & $2.11 \mathrm{E}-04$ & $1.77 \mathrm{E}+05$ & $2.78 \mathrm{E}+05$ & -3.92 & -3.71 & 0.024 \\
\hline & & & & 28161 & (o) & 3 & 216.045 & $1.07 \mathrm{E}-02$ & $8.94 \mathrm{E}+06$ & $1.40 \mathrm{E}+07$ & -2.21 & -2.01 & 0.455 \\
\hline & & & & 29742 & (o) & 1 & 223.689 & $5.46 \mathrm{E}-04$ & $4.58 \mathrm{E}+05$ & $7.19 \mathrm{E}+05$ & -3.47 & -3.27 & 0.005 \\
\hline & & & & 29824 & (o) & 2 & 224.099 & $2.39 \mathrm{E}-05$ & $2.00 \mathrm{E}+04$ & $3.14 \mathrm{E}+04$ & -4.83 & -4.63 & 0.001 \\
\hline & & & & 30816 & (o) & 1 & 229.195 & $4.05 \mathrm{E}-03$ & $3.40 \mathrm{E}+06$ & $5.34 \mathrm{E}+06$ & -2.58 & -2.38 & 0.002 \\
\hline & & & & 32350 & (o) & 3 & 237.551 & $8.23 \mathrm{E}-01$ & $6.90 \mathrm{E}+08$ & $1.08 \mathrm{E}+09$ & -0.24 & -0.04 & 0.251 \\
\hline & & & & 39115 & (o) & 1 & 283.056 & $2.83 \mathrm{E}-03$ & $2.37 \mathrm{E}+06$ & $3.72 \mathrm{E}+06$ & -2.56 & -2.35 & 0.244 \\
\hline & & & & 39346 & (o) & 2 & 284.916 & $1.05 \mathrm{E}-02$ & $8.83 \mathrm{E}+06$ & $1.39 \mathrm{E}+07$ & -1.98 & -1.77 & 0.660 \\
\hline & & & & 55715 & (o) & 1 & 534.098 & $4.13 \mathrm{E}-04$ & $3.46 \mathrm{E}+05$ & $5.43 \mathrm{E}+05$ & -2.85 & -2.63 & 0.000 \\
\hline & & & & 66048 & (o) & 2 & 1192.292 & $3.94 \mathrm{E}-02$ & $3.30 \mathrm{E}+07$ & $5.18 \mathrm{E}+07$ & -0.20 & 0.04 & 0.693 \\
\hline & & & & 66390 & (o) & 1 & 1242.891 & $4.01 \mathrm{E}-05$ & $3.36 \mathrm{E}+04$ & $5.27 \mathrm{E}+04$ & -3.17 & -2.91 & 0.227 \\
\hline & & & & 66493 & (o) & 2 & 1259.000 & $6.15 \mathrm{E}-05$ & $5.16 \mathrm{E}+04$ & $8.10 \mathrm{E}+04$ & -2.97 & -2.72 & 0.112 \\
\hline & & & & 27444 & (o) & 2 & 204.362 & $3.68 \mathrm{E}-04$ & $9.44 \mathrm{E}+05$ & $9.70 \mathrm{E}+05$ & -3.23 & -3.22 & 0.387 \\
\hline & & & & 27918 & (o) & 1 & 206.362 & $7.57 \mathrm{E}-02$ & $1.94 \mathrm{E}+08$ & $1.99 \mathrm{E}+08$ & -0.91 & -0.90 & 0.507 \\
\hline & & & & 28021 & (o) & 2 & 206.804 & $2.30 \mathrm{E}-01$ & $5.88 \mathrm{E}+08$ & $6.04 \mathrm{E}+08$ & -0.42 & -0.41 & 0.527 \\
\hline & & & & 29736 & (o) & 0 & 214.412 & $9.25 \mathrm{E}-05$ & $2.37 \mathrm{E}+05$ & $2.43 \mathrm{E}+05$ & -3.79 & -3.77 & 0.000 \\
\hline & & & & 29742 & (o) & 1 & 214.439 & $2.71 \mathrm{E}-04$ & $6.93 \mathrm{E}+05$ & $7.12 \mathrm{E}+05$ & -3.32 & -3.31 & 0.002 \\
\hline & & & & 29824 & (o) & 2 & 214.816 & $5.31 \mathrm{E}-05$ & $1.36 \mathrm{E}+05$ & $1.40 \mathrm{E}+05$ & -4.03 & -4.01 & 0.000 \\
\hline & & & & 30816 & (o) & 1 & 219.494 & $9.17 \mathrm{E}-05$ & $2.35 \mathrm{E}+05$ & $2.41 \mathrm{E}+05$ & -3.77 & -3.76 & 0.015 \\
\hline & & & & 39002 & (o) & 0 & 267.597 & $2.33 \mathrm{E}-01$ & $5.96 \mathrm{E}+08$ & $6.12 \mathrm{E}+08$ & -0.19 & -0.18 & 0.708 \\
\hline & & & & 39115 & (o) & 1 & 268.407 & $1.73 \mathrm{E}-01$ & $4.44 \mathrm{E}+08$ & $4.56 \mathrm{E}+08$ & -0.32 & -0.31 & 0.705 \\
\hline & & & & 39346 & (o) & 2 & 270.079 & $2.84 \mathrm{E}-01$ & $7.28 \mathrm{E}+08$ & $7.48 \mathrm{E}+08$ & -0.10 & -0.09 & 0.709 \\
\hline & & & & 55715 & (o) & 1 & 484.233 & $1.12 \mathrm{E}-06$ & $2.88 \mathrm{E}+03$ & $2.96 \mathrm{E}+03$ & -5.00 & -4.98 & 0.080 \\
\hline & & & & 66048 & (o) & 2 & 969.440 & $1.10 \mathrm{E}-05$ & $2.83 \mathrm{E}+04$ & $2.91 \mathrm{E}+04$ & -3.40 & -3.39 & 0.091 \\
\hline & & & & 66390 & (o) & 1 & 1002.628 & $2.87 \mathrm{E}-04$ & $7.35 \mathrm{E}+05$ & $7.55 \mathrm{E}+05$ & -1.96 & -1.94 & 0.402 \\
\hline & & & & 66493 & (o) & 2 & 1013.085 & $4.41 \mathrm{E}-04$ & $1.13 \mathrm{E}+06$ & $1.16 \mathrm{E}+06$ & -1.76 & -1.75 & 0.319 \\
\hline & & & & 66460 & (o) & 2 & 1009.706 & $1.72 \mathrm{E}-04$ & $4.40 \mathrm{E}+05$ & $4.52 \mathrm{E}+05$ & -2.17 & -2.16 & 0.307 \\
\hline & & & & 67237 & (o) & 0 & 1095.698 & $9.72 \mathrm{E}-04$ & $2.49 \mathrm{E}+06$ & $2.56 \mathrm{E}+06$ & -1.35 & -1.34 & 0.575 \\
\hline & & & & 67298 & (o) & 1 & 1103.071 & $6.87 \mathrm{E}-04$ & $1.76 \mathrm{E}+06$ & $1.81 \mathrm{E}+06$ & -1.49 & -1.48 & 0.528 \\
\hline & & & & 67396 & (o) & 2 & 1115.192 & $9.76 \mathrm{E}-04$ & $2.50 \mathrm{E}+06$ & $2.57 \mathrm{E}+06$ & -1.33 & -1.32 & 0.472 \\
\hline & & & & 68498 & (o) & 1 & 1271.473 & $3.72 \mathrm{E}-07$ & $9.52 \mathrm{E}+02$ & $9.78 \mathrm{E}+02$ & -4.64 & -4.63 & 0.029 \\
\hline & & & & 75308 & (o) & 2 & 9497.064 & $1.05 \mathrm{E}-07$ & $2.69 \mathrm{E}+02$ & $2.76 \mathrm{E}+02$ & -3.44 & -3.43 & 0.567 \\
\hline & & & & 75591 & (o) & 2 & 12984.147 & $3.27 \mathrm{E}-07$ & $8.37 \mathrm{E}+02$ & $8.60 \mathrm{E}+02$ & -2.68 & -2.66 & 0.581 \\
\hline & & & & 75651 & (o) & 1 & 14082.652 & $2.29 \mathrm{E}-07$ & $5.87 \mathrm{E}+02$ & $6.03 \mathrm{E}+02$ & -2.76 & -2.75 & 0.295 \\
\hline & & & & 75681 & (o) & 2 & 14699.495 & $9.76 \mathrm{E}-07$ & $2.50 \mathrm{E}+03$ & $2.57 \mathrm{E}+03$ & -2.10 & -2.08 & 0.578 \\
\hline & & & & 75913 & (o) & 2 & 22304.392 & $1.26 \mathrm{E}-08$ & $3.22 \mathrm{E}+01$ & $3.31 \mathrm{E}+01$ & -3.64 & -3.61 & 0.035 \\
\hline
\end{tabular}


Table 6 - continued

\begin{tabular}{|c|c|c|c|c|c|c|c|c|c|c|c|c|c|}
\hline \multicolumn{3}{|c|}{ Upper level $^{a}$} & $\tau_{u}(\mathrm{~ns})$ & \multicolumn{3}{|c|}{ Lower level $^{a}$} & $\frac{\lambda^{b}(\mathrm{~nm})}{27287.372}$ & $\frac{B F}{2.90 \mathrm{E}-08}$ & $\frac{g A\left(\mathrm{~s}^{-1}\right)}{7.44 \mathrm{E}+01}$ & $\frac{g A_{\text {resc }}\left(\mathrm{s}^{-1}\right)}{7.64 \mathrm{E}+01}$ & $\begin{array}{r}\log (g f) \\
-3.11\end{array}$ & $\begin{array}{c}\log (g f)_{\text {resc }} \\
-3.07\end{array}$ & $\frac{\mathrm{CF}^{c}}{0.363}$ \\
\hline & & & & 76073 & (o) & 1 & 34770.709 & $1.85 \mathrm{E}-10$ & $4.75 \mathrm{E}-01$ & $4.88 \mathrm{E}-01$ & -5.04 & -5.05 & 0.031 \\
\hline & & & & 27444 & (o) & 2 & 203.412 & $7.58 \mathrm{E}-05$ & $3.20 \mathrm{E}+05$ & $3.48 \mathrm{E}+05$ & -3.70 & -3.67 & 0.170 \\
\hline & & & & 27602 & (o) & 3 & 204.071 & $1.10 \mathrm{E}-03$ & $4.64 \mathrm{E}+06$ & $5.04 \mathrm{E}+06$ & -2.54 & -2.50 & 0.490 \\
\hline & & & & 27918 & (o) & 1 & 205.393 & $3.08 \mathrm{E}-03$ & $1.30 \mathrm{E}+07$ & $1.41 \mathrm{E}+07$ & -2.08 & -2.05 & 0.390 \\
\hline & & & & 28021 & (o) & 2 & 205.831 & $4.50 \mathrm{E}-02$ & $1.90 \mathrm{E}+08$ & $2.07 \mathrm{E}+08$ & -0.92 & -0.88 & 0.454 \\
\hline & & & & 29742 & (o) & 1 & 213.393 & $1.33 \mathrm{E}-05$ & $5.60 \mathrm{E}+04$ & $6.09 \mathrm{E}+04$ & -4.42 & -4.38 & 0.000 \\
\hline & & & & 29824 & (o) & 2 & 213.766 & $4.74 \mathrm{E}-04$ & $2.00 \mathrm{E}+06$ & $2.17 \mathrm{E}+06$ & -2.86 & -2.83 & 0.001 \\
\hline & & & & 30816 & (o) & 1 & 218.398 & $1.25 \mathrm{E}-04$ & $5.27 \mathrm{E}+05$ & $5.73 \mathrm{E}+05$ & -3.42 & -3.39 & 0.012 \\
\hline & & & & 32350 & (o) & 3 & 225.973 & $1.25 \mathrm{E}-03$ & $5.26 \mathrm{E}+06$ & $5.72 \mathrm{E}+06$ & -2.39 & -2.36 & 0.328 \\
\hline & & & & 39115 & (o) & 1 & 266.771 & $1.77 \mathrm{E}-01$ & $7.46 \mathrm{E}+08$ & $8.11 \mathrm{E}+08$ & -0.10 & -0.06 & 0.705 \\
\hline & & & & 39346 & (o) & 2 & 268.422 & $5.17 \mathrm{E}-01$ & $2.18 \mathrm{E}+09$ & $2.37 \mathrm{E}+09$ & 0.37 & 0.41 & 0.709 \\
\hline & & & & 55715 & (o) & 1 & 478.932 & $2.35 \mathrm{E}-05$ & $9.91 \mathrm{E}+04$ & $1.08 \mathrm{E}+05$ & -3.47 & -3.43 & 0.019 \\
\hline & & & & 66564 & (o) & 3 & 997.176 & $6.97 \mathrm{E}-04$ & $2.94 \mathrm{E}+06$ & $3.20 \mathrm{E}+06$ & -1.36 & -1.32 & 0.372 \\
\hline & & & & 66584 & (o) & 3 & 999.182 & $1.61 \mathrm{E}-04$ & $6.78 \mathrm{E}+05$ & $7.37 \mathrm{E}+05$ & -1.99 & -1.96 & 0.215 \\
\hline & & & & 67298 & (o) & 1 & 1075.944 & $8.96 \mathrm{E}-04$ & $3.78 \mathrm{E}+06$ & $4.11 \mathrm{E}+06$ & -1.18 & -1.15 & 0.593 \\
\hline & & & & 67396 & (o) & 2 & 1087.473 & $2.06 \mathrm{E}-03$ & $8.71 \mathrm{E}+06$ & $9.47 \mathrm{E}+06$ & -0.81 & -0.77 & 0.517 \\
\hline & & & & 67744 & (o) & 3 & 1130.199 & $9.83 \mathrm{E}-05$ & $4.15 \mathrm{E}+05$ & $4.51 \mathrm{E}+05$ & -2.10 & -2.06 & 0.549 \\
\hline & & & & 68498 & (o) & 1 & 1235.566 & $1.70 \mathrm{E}-05$ & $7.17 \mathrm{E}+04$ & $7.79 \mathrm{E}+04$ & -2.78 & -2.75 & 0.138 \\
\hline & & & & 75308 & (o) & 2 & 7803.238 & $4.76 \mathrm{E}-08$ & $2.01 \mathrm{E}+02$ & $2.18 \mathrm{E}+02$ & -3.74 & -3.70 & 0.536 \\
\hline & & & & 75309 & (o) & 3 & 7806.711 & $2.44 \mathrm{E}-07$ & $1.03 \mathrm{E}+03$ & $1.12 \mathrm{E}+03$ & -3.03 & -2.99 & 0.670 \\
\hline & & & & 75373 & (o) & 3 & 8220.091 & $2.89 \mathrm{E}-07$ & $1.22 \mathrm{E}+03$ & $1.33 \mathrm{E}+03$ & -2.91 & -2.87 & 0.502 \\
\hline & & & & 75552 & (o) & 3 & 9642.061 & $1.18 \mathrm{E}-07$ & $5.00 \mathrm{E}+02$ & $5.43 \mathrm{E}+02$ & -3.16 & -3.12 & 0.304 \\
\hline & & & & 75591 & (o) & 2 & 10012.694 & $8.81 \mathrm{E}-09$ & $3.72 \mathrm{E}+01$ & $4.04 \mathrm{E}+01$ & -4.26 & -4.22 & 0.012 \\
\hline & & & & 75651 & (o) & 1 & 10653.532 & $2.30 \mathrm{E}-09$ & $9.70 \mathrm{E}+00$ & $1.05 \mathrm{E}+01$ & -4.79 & -4.75 & 0.011 \\
\hline & & & & 27841 & (o) & 4 & 201.768 & $2.02 \mathrm{E}-01$ & $2.56 \mathrm{E}+08$ & $3.80 \mathrm{E}+08$ & -0.80 & -0.63 & 0.327 \\
\hline & & & & 28021 & (o) & 2 & 202.504 & $1.99 \mathrm{E}-02$ & $2.52 \mathrm{E}+07$ & $3.74 \mathrm{E}+07$ & -1.81 & -1.64 & 0.293 \\
\hline & & & & 28161 & (o) & 3 & 203.079 & $1.29 \mathrm{E}-01$ & $1.63 \mathrm{E}+08$ & $2.42 \mathrm{E}+08$ & -0.99 & -0.82 & 0.269 \\
\hline & & & & 29824 & (o) & 2 & 210.180 & $1.76 \mathrm{E}-01$ & $2.23 \mathrm{E}+08$ & $3.31 \mathrm{E}+08$ & -0.83 & -0.66 & 0.374 \\
\hline & & & & 32350 & (o) & 3 & 221.970 & $4.87 \mathrm{E}-05$ & $6.16 \mathrm{E}+04$ & $9.14 \mathrm{E}+04$ & -4.34 & -4.17 & 0.126 \\
\hline & & & & 39346 & (o) & 2 & 262.791 & $1.16 \mathrm{E}-01$ & $1.47 \mathrm{E}+08$ & $2.18 \mathrm{E}+08$ & -0.81 & -0.65 & 0.249 \\
\hline & & & & 66048 & (o) & 2 & 881.687 & $2.62 \mathrm{E}-04$ & $3.32 \mathrm{E}+05$ & $4.93 \mathrm{E}+05$ & -2.39 & -2.24 & 0.123 \\
\hline & & & & 66493 & (o) & 2 & 917.642 & $8.86 \mathrm{E}-03$ & $1.12 \mathrm{E}+07$ & $1.66 \mathrm{E}+07$ & -0.83 & -0.68 & 0.509 \\
\hline & & & & 66460 & (o) & 2 & 914.869 & $6.78 \mathrm{E}-03$ & $8.58 \mathrm{E}+06$ & $1.27 \mathrm{E}+07$ & -0.95 & -0.80 & 0.781 \\
\hline & & & & 66564 & (o) & 3 & 923.667 & $6.02 \mathrm{E}-02$ & $7.62 \mathrm{E}+07$ & $1.13 \mathrm{E}+08$ & 0.01 & 0.16 & 0.379 \\
\hline & & & & 66584 & (o) & 3 & 925.388 & $7.61 \mathrm{E}-02$ & $9.63 \mathrm{E}+07$ & $1.43 \mathrm{E}+08$ & 0.11 & 0.26 & 0.792 \\
\hline & & & & 66719 & (o) & 4 & 937.110 & $1.33 \mathrm{E}-01$ & $1.68 \mathrm{E}+08$ & $2.49 \mathrm{E}+08$ & 0.36 & 0.52 & 0.748 \\
\hline & & & & 67396 & (o) & 2 & 1000.629 & $4.64 \mathrm{E}-02$ & $5.87 \mathrm{E}+07$ & $8.71 \mathrm{E}+07$ & -0.04 & 0.12 & 0.575 \\
\hline & & & & 67744 & (o) & 3 & 1036.689 & $4.70 \mathrm{E}-05$ & $5.94 \mathrm{E}+04$ & $8.81 \mathrm{E}+04$ & -3.00 & -2.85 & 0.115 \\
\hline & & & & 75221 & (o) & 4 & 4616.186 & $1.04 \mathrm{E}-06$ & $1.31 \mathrm{E}+03$ & $1.94 \mathrm{E}+03$ & -3.28 & -3.21 & 0.152 \\
\hline & & & & 75308 & (o) & 2 & 4808.601 & $2.30 \mathrm{E}-08$ & $2.91 \mathrm{E}+01$ & $4.32 \mathrm{E}+01$ & -4.90 & -4.82 & 0.068 \\
\hline & & & & 75309 & (o) & 3 & 4809.920 & $4.80 \mathrm{E}-08$ & $6.07 \mathrm{E}+01$ & $9.01 \mathrm{E}+01$ & -4.58 & -4.50 & 0.011 \\
\hline & & & & 75373 & (o) & 3 & 4963.717 & $4.93 \mathrm{E}-07$ & $6.24 \mathrm{E}+02$ & $9.26 \mathrm{E}+02$ & -3.54 & -3.47 & 0.150 \\
\hline & & & & 75390 & (o) & 4 & 5006.948 & $1.42 \mathrm{E}-06$ & $1.80 \mathrm{E}+03$ & $2.67 \mathrm{E}+03$ & -3.07 & -3.00 & 0.104 \\
\hline & & & & 75470 & (o) & 4 & 5215.252 & $1.24 \mathrm{E}-06$ & $1.57 \mathrm{E}+03$ & $2.33 \mathrm{E}+03$ & -3.09 & -3.02 & 0.112 \\
\hline & & & & 75552 & (o) & 3 & 5448.967 & $1.03 \mathrm{E}-06$ & $1.30 \mathrm{E}+03$ & $1.93 \mathrm{E}+03$ & -3.13 & -3.07 & 0.538 \\
\hline & & & & 75561 & (o) & 4 & 5474.449 & $1.18 \mathrm{E}-08$ & $1.49 \mathrm{E}+01$ & $2.21 \mathrm{E}+01$ & -5.05 & -5.00 & 0.107 \\
\hline & & & & 75591 & (o) & 2 & 5565.388 & $5.53 \mathrm{E}-07$ & $7.00 \mathrm{E}+02$ & $1.04 \mathrm{E}+03$ & -3.37 & -3.32 & 0.306 \\
\hline
\end{tabular}


Table 6 - continued

\begin{tabular}{|c|c|c|c|c|c|c|c|c|c|c|c|}
\hline \multirow[t]{4}{*}{ Upper level $^{a}$} & \multirow[t]{4}{*}{$\tau_{u}(\mathrm{~ns})$} & \multicolumn{3}{|c|}{ Lower level $^{a}$} & \multirow{2}{*}{$\frac{\lambda^{b}(\mathrm{~nm})}{5858.418}$} & \multirow{2}{*}{$\frac{B F}{1.88 \mathrm{E}-06}$} & \multirow{2}{*}{$\frac{g A\left(\mathrm{~s}^{-1}\right)}{2.38 \mathrm{E}+03}$} & \multirow{2}{*}{$\frac{g A_{\text {resc }}\left(\mathrm{s}^{-1}\right)}{3.53 \mathrm{E}+03}$} & \multirow{2}{*}{$\begin{array}{r}\log (g f) \\
-2.79\end{array}$} & \multirow{2}{*}{$\frac{\log (g f)_{\text {resc }}}{-2.74}$} & \multirow{2}{*}{$\frac{\mathrm{CF}^{c}}{0.571}$} \\
\hline & & 75681 & (o) & 2 & & & & & & & \\
\hline & & 75716 & (o) & 3 & 5981.306 & $1.01 \mathrm{E}-05$ & $1.28 \mathrm{E}+04$ & $1.90 \mathrm{E}+04$ & -2.04 & -1.99 & 0.848 \\
\hline & & 75913 & (o) & 2 & 6779.698 & $4.40 \mathrm{E}-07$ & $5.56 \mathrm{E}+02$ & $8.25 \mathrm{E}+02$ & -3.28 & -3.24 & 0.072 \\
\hline
\end{tabular}

Notes. ${ }^{a}$ Each level is designated by its value in $\mathrm{cm}^{-1}$, its parity ((e) and (o) stand for even and odd, respectively) and its total quantum number, $J$.

${ }^{b}$ Calculated from the energy levels compiled by NIST (Kramida et al. 2015). $\lambda>200 \mathrm{~nm}$ are given in air.

${ }^{c}$ Cancellation factor as defined by Cowan (1981). A value less than 5 percent indicates a strong cancellation effect on the line strength and the transition probability could be underestimated.

${ }^{d}$ TR-LIF measurements by Marsden et al. (1988).

${ }^{e}$ TR-LIF measurements, this work.

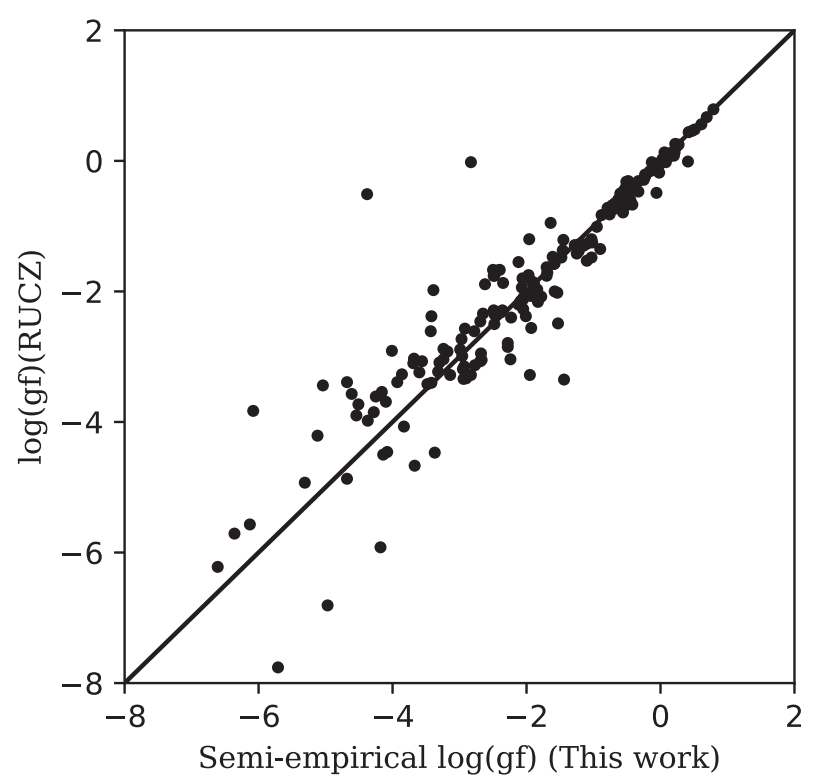

Figure 4. A comparison between the oscillator strengths determined by the combination of the HFR+CPOL branching fractions and the TR-LIF lifetimes (this work) and the semi-empirical oscillator strengths calculated by Ruczkowski et al. (2014) (RUCZ). The straight line of equality has been drawn.

the meteoritic abundances (Lodders et al. 2009) still remains for scandium with $\log \epsilon_{\odot}=3.16 \pm 0.04$ and $\log \epsilon_{\text {met }}=3.05 \pm 0.02$.

The Sc II lines used in Scott et al. (2015) for the determination of the solar abundance of scandium are presented in Table 7 . These lines are from low-excited levels measured by Lawler \& Dakin (1989) but not included in this study. The third column of this table contains the oscillator strengths deduced from the $A$-values of Lawler \& Dakin (1989) used by Scott et al. (2015) to determine the photospheric abundances listed in the sixth column. They are compared to our rescaled oscillator strengths reported in the fourth column and the differences between the two values are given in the log scale in the fifth column. For this set of solar lines, our oscillator strengths are systematically larger than those of Lawler \& Dakin (1989) by $\sim 0.1$ dex on average, if we exclude the transition $3 \mathrm{~d}^{2}{ }^{1} \mathrm{D}_{2}-3 \mathrm{~d} 4 \mathrm{p}{ }^{1} \mathrm{D}_{2}^{\mathrm{o}}$ for which our $f$-value is affected by strong cancellation effects. Column seven in Table 7 gives the abundances obtained from each line, assuming they are all lying on the linear part of the curve of growth (see the upper left panel of fig. 3 in Scott et al. (2015)), with our new $g$-values.

The weighted average along with the corresponding weighted standard deviation of the abundance was determined using the weights of Scott et al. (2015), reported in the last column of

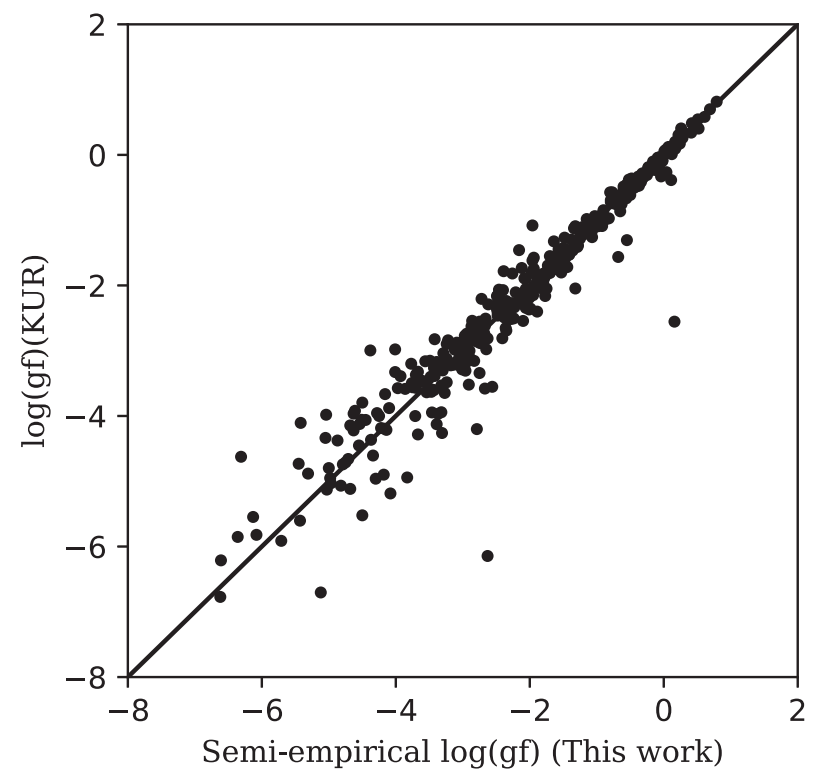

Figure 5. A comparison between the oscillator strengths determined by the combination of the HFR+CPOL branching fractions and the TR-LIF lifetimes (this work) and the oscillator strengths calculated by Kurucz (2011) (KUR). The straight line of equality has been drawn.

Table 7. Their weights range from one to three and are based on the line quality for abundance determination. Discarding the line $3 d^{2}{ }^{1} D_{2}-3 d 4 p{ }^{1} D_{2}^{o}$ from the mean estimate, one obtains $\log \epsilon_{\text {cor }}=3.04 \pm 0.13$ (where the second number is the standard deviation) for the corrected photospheric abundance, now in good agreement with the meteoritic value of Lodders et al. (2009). Even if we reject the transition $3 d^{2}{ }^{3} F_{4}-3 d 4 p{ }^{3} F_{3}^{o}$ for which there is a factor of two difference between our rescaled $f$-value and the experimental value of Lawler \& Dakin (1989), the mean $\log \epsilon_{\text {cor }}=3.10 \pm 0.05$ is still in accord with the meteoritic value. Moreover, considering the full line set does not change the agreement $\left(\log \epsilon_{\text {cor }}=3.07 \pm 0.17\right)$. Finally, we note that all these weighted average abundances agree within the mutual error bars with the value determined by Scott et al. (2015) using only Sc I lines $(\log \epsilon=3.14 \pm 0.09)$.

Replacing our $f$-value set by the one of Kurucz (2011) will not change this accord either $\left(\log \epsilon_{\text {kur }}=3.10 \pm 0.09\right)$. This is not the case for the set of Ruczkowski et al. (2014). Indeed, the photospheric abundance would be estimated significantly too high with respect to the meteoritic value, i.e. $\log \epsilon_{\text {ruc }}=3.44 \pm 0.31$. Even if the transition $3 d^{2}{ }^{3} F_{4}-3 d 4 p^{3} F_{3}^{o}$ for which the oscillator strength calculated by Ruczkowski et al. (2014) $\left(\log (g f)_{\text {ruc }}=-3.28\right)$ is one order of magnitude lower than the experimental value of Lawler \& 
Table 7. Sc II lines used in the determination of the solar abundance $(\log \epsilon)$ of scandium.

\begin{tabular}{|c|c|c|c|c|c|c|c|c|c|}
\hline \multicolumn{3}{|c|}{ Transition } & \multirow{2}{*}{$\frac{\lambda^{a}(\mathrm{~nm})}{442.067}$} & \multirow{2}{*}{$\frac{\log (g f)_{\mathrm{L} \& \mathrm{D}}^{b}}{-2.273}$} & \multirow{2}{*}{$\frac{\log (g f)_{\mathrm{resc}}^{c}}{-1.950}$} & \multirow{2}{*}{$\frac{\Delta \log (g f)^{d}}{0.323}$} & \multirow{2}{*}{$\frac{\log \epsilon^{e}}{3.099}$} & \multirow{2}{*}{$\frac{\log \epsilon_{\mathrm{cor}}^{f}}{2.776}$} & \multirow{2}{*}{$\frac{\text { Weight }^{e}}{2}$} \\
\hline $3 d^{2}{ }^{3} F_{4}$ & - & $3 \mathrm{~d} 4 \mathrm{p}^{3} \mathrm{~F}_{3}^{\mathrm{o}}$ & & & & & & & \\
\hline $3 d^{2}{ }^{3} F_{3}$ & - & $3 \mathrm{~d} 4 \mathrm{p}^{3} \mathrm{~F}_{2}^{\mathrm{o}}$ & 443.135 & -1.969 & -1.780 & 0.189 & 3.155 & 2.966 & 1 \\
\hline $3 d^{23} P_{2}$ & - & $3 \mathrm{~d} 4 \mathrm{p}^{1} \mathrm{P}_{1}^{\mathrm{o}}$ & 535.720 & -2.111 & -2.050 & 0.061 & 3.131 & 3.070 & 2 \\
\hline $3 d^{23} P_{1}$ & - & $3 \mathrm{~d} 4 \mathrm{p}^{3} \mathrm{P}_{2}^{\mathrm{o}}$ & 564.100 & -1.131 & -1.000 & 0.131 & 3.226 & 3.095 & 1 \\
\hline $3 d^{2}{ }^{3} P_{0}$ & - & $3 \mathrm{~d} 4 \mathrm{p}^{3} \mathrm{P}_{1}^{\mathrm{o}}$ & 565.836 & -1.208 & -1.150 & 0.058 & 3.211 & 3.153 & 1 \\
\hline $3 d^{2}{ }^{3} P_{1}$ & - & $3 \mathrm{~d} 4 \mathrm{p}^{3} \mathrm{P}_{1}^{\mathrm{o}}$ & 566.715 & -1.309 & -1.220 & 0.089 & 3.235 & 3.146 & 1 \\
\hline $3 d^{23} P_{1}$ & - & $3 \mathrm{~d} 4 \mathrm{p}^{3} \mathrm{P}_{0}^{\mathrm{o}}$ & 566.904 & -1.200 & -1.100 & 0.100 & 3.246 & 3.146 & 1 \\
\hline $3 d^{2}{ }^{3} P_{2}$ & - & $3 \mathrm{~d} 4 \mathrm{p}^{3} \mathrm{P}_{1}^{\mathrm{o}}$ & 568.420 & -1.074 & -1.030 & 0.044 & 3.154 & 3.110 & 2 \\
\hline $3 d^{21} D_{2}$ & - & $3 d 4 p^{1} D_{2}^{o}$ & 660.460 & -1.309 & $-1.570^{g}$ & -0.261 & 3.204 & 3.465 & 1 \\
\hline
\end{tabular}

Notes. ${ }^{a}$ Calculated from the energy levels compiled by NIST (Kramida et al. 2015).

${ }^{b}$ Scott et al. (2015), deduced from the A-values of Lawler \& Dakin (1989).

${ }^{c}$ This work, rescaled value.

${ }^{d} \Delta \log (g f)=\log (g f)_{\mathrm{L} \& \mathrm{D}}-\log (g f)_{\text {resc }}$.

${ }^{e}$ Scott et al. (2015).

${ }^{f}$ This work, corrected abundance, $\log \epsilon_{\text {cor }}=\log \epsilon-\Delta \log (g f)$.

${ }^{g}$ Affected by strong cancellation effects $(C F<0.05)$.

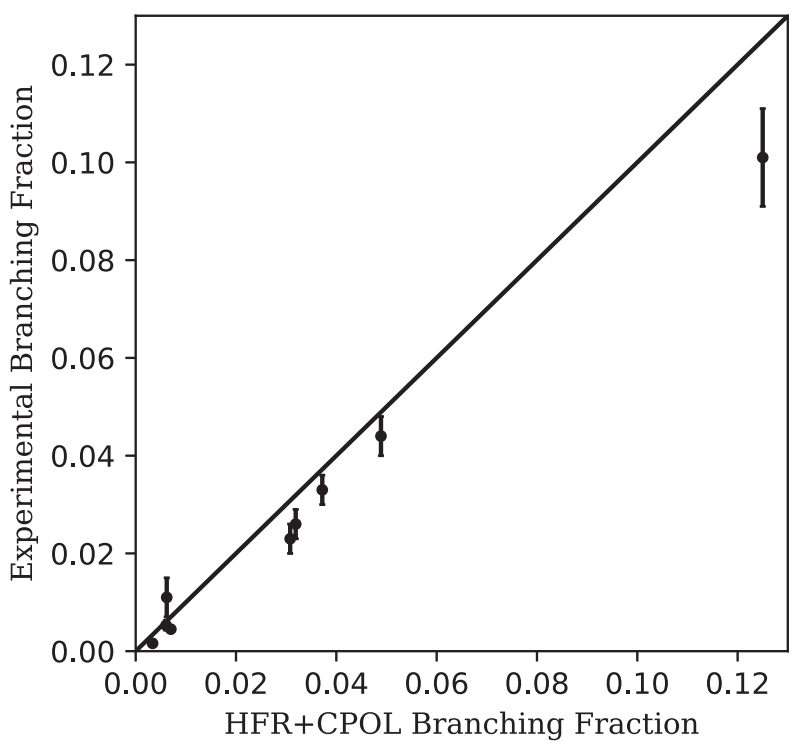

Figure 6. A comparison between the HFR+CPOL branching fractions and the experimental values of Lawler \& Dakin (1989) for the Sc II lines used in the determination of the solar scandium abundance. The straight line of equality has been drawn.

Dakin (1989) is excluded, this would not significantly improve the situation $\left(\log \epsilon_{\text {ruc }}=3.29 \pm 0.01\right)$.

It should be noted, however, that the lines used for these studies are weak, see Fig. 6. Their $B F s$ are less than 5 per cent except for $\lambda 566.904$ having $\sim 10$ percent. These small BFs make it difficult to measure and calculate with high accuracy. The real uncertainty might thus be larger than the observed scatter.

\section{CONCLUSIONS}

New TR-LIF lifetimes were measured using two-step excitation schemes in $\mathrm{Sc}$ II. These measurements extend the set of available experimental values with 17 even-parity levels belonging to the excited configurations $3 \mathrm{~d} 5 \mathrm{~s}, 3 \mathrm{~d} 4 \mathrm{~d}, 4 \mathrm{p}^{2}$ and $3 \mathrm{~d} 6 \mathrm{~s}$. We measured 57 $B F s$ from these upper levels using an HCL and an FTS. By combining the $B F s$ with the measured lifetimes, we derived $\log (g f)$ values from these highly excited levels. A HFR model that includes the main relativistic interactions along with the core-polarization effects (HFR+CPOL) was used to determine the branching fractions and the oscillator strengths. The comparison between our HFR+CPOL and TR-LIF lifetimes along with those found in the literature (Buchta et al. 1971; Arnesen et al. 1976; Palenius et al. 1976; Vogel et al. 1985; Marsden et al. 1988; Kurucz 2011; Ruczkowski et al. 2014) shows generally a good agreement ranging from a few per cent to 20 percent with the notable exceptions of the evenparity levels $4 \mathrm{p}^{2}{ }^{1} \mathrm{D}_{2}$ and $3 \mathrm{~d} 6 \mathrm{~s}^{3} \mathrm{D}_{3}$. The former discrepancy may be due to a cancellation effect that lengthens the HFR+CPOL lifetime. Owing to the good agreement ( $\sim 20$ per cent) obtained with the experimental branching fractions of Lawler \& Dakin (1989) for low-excitation levels and ours for high-excitation levels, the HFR+CPOL branching fractions were combined with our TR-LIF lifetimes and the experimental values of Marsden et al. (1988) to obtain rescaled semi-empirical oscillator strengths for all the 380 E1 transitions depopulating the 34 fine-structure levels for which TR-LIF lifetimes are available. This new set of oscillator strengths was compared to the parametric calculation of Ruczkowski et al. (2014) and to the HFR values of Kurucz (2011). In both cases, the mean scatters were $\sim 20$ percent for the strong lines $(\log (g f)$ $\gtrsim-1)$ giving an estimate of the accuracy for these radiative parameters. Finally, the solar abundance of scandium was estimated to $\log \epsilon \odot=3.04 \pm 0.13$ using our rescaled semi-empirical oscillator strengths to correct the values determined in the recent study of Scott et al. (2015). This value is in improved agreement with the meteoritic value ( $\left.\log \epsilon_{\mathrm{met}}=3.05 \pm 0.02\right)$ of Lodders et al. (2009).

\section{ACKNOWLEDGEMENTS}

This work was financially supported by the Integrated Initiative of Infrastructure Project LASERLAB-EUROPE, contract LLC002130 and the Belgian FRS-FNRS. PQ and PP are Research Director and Research Associate of the FRS-FNRS, respectively. We acknowledge the support from the Swedish Research Council through a Linnaeus grant to the Lund Laser Centre and through project grant 2016-04185, as well as the Knut and Alice Wallenberg Foundation. MTB, JCP and CC thank the STFC (UK) for support of their Laboratory Astrophysics research at Imperial College London. VF is currently a post-doctoral researcher of the Return Grant programme of the Belgian Scientific Policy (BELSPO). The Belgian team is grateful to the Swedish colleagues for the warm 
hospitality enjoyed at the Lund Laser Centre during the two campaigns of measurements performed in 2015 June and August.

\section{REFERENCES}

Arnesen A., Bengtsson B., Curtis L. J., Hallin R., Nordling C., Noreland T., 1976, Phys. Lett., 56A, 355

Buchta R., Curtis L. J., Martinson I., Brzozowski J., 1971, Phys. Scr., 4, 55

Cowan R. D., 1981, The Theory of Atomic Structure and Spectra. University of California Press, Berkeley

Davis S. P., Abrams M. C., Brault J. W., 2001, Fourier Transform Spectrometry. Academic Press, San Diego

Engström L., 1998, Technical Report LRAP-232, A Computer Program to Determine Peak Positions and Intensities in Experimental Spectra. Atomic Physics, Lund Univ., Lund, Sweden

Engström L., 2014, GFit. Available at: http://kurslab-atom.fysik.lth.se/ Lars/GFit/Html/index.html

Engström L., Lundberg H., Nilsson H., Hartman H., Bäckström E., 2014, A\&A, 570, A34

Johansson S., Litzén U., 1980, Phys. Scr., 22, 49

Johnson W. R., Kolb D., Huang K.-N., 1983, Atom. Dat. Nucl. Dat. Tables, 28,333

Kramida A., Ralchenko Yu., Reader J., NIST ASD Team, 2015, http:// physics.nist.gov/asd (accessed 2015 August 17)

Kurucz R. L., 2011, http://kurucz.harvard.edu/atoms.html (accessed 2015 August 17)

Lawler J. E., Dakin J. T., 1989, J. Opt. Soc. Am., 6, 1457

Lind K., Bergmann M., Asplund M., 2012, MNRAS, 427, 50
Lodders K., Palme H., Gail H.-P., 2009, in Trümper J. E., ed., Landolt Börnstein, New Series, Vol. VI/4B, Chap. 4.4, Abundances of the Elements in the Solar System, Springer-Verlag, Berlin, p. 560

Lundberg H. et al., 2016, MNRAS, 460, 356

Marsden G. C., Den Hartog E. A., Lawler J. E., Dakin J. T., Roberts V. D., 1988, J. Opt. Soc. Am., 5, 606

Neufeld L. W., 1970, Dissertation, Kansas State Univ.

Pagel E. B. J., 2009, Nucleosynthesis and Chemical Evolution of Galaxies. Cambridge Univ. Press, Cambridge

Palenius H. P., Curtis L. J., Lundlin L., 1976, J. Phys. B, 9, L473

Palmeri P., Quinet P., Fivet V., Biémont E., Nilsson H., Engström L., Lundberg H., 2008, Phys. Scr., 78, 015304

Pehlivan A., Nilsson H., Hartman H., 2015, A\&A, 582, A98

Pickering J. C., 2002, Vib. Spectrosc., 29(1,2), 27

Quinet P., Palmeri P., Biémont E., McCurdy M. M., Rieger G., Pinnington E. H., Wickliffe M. E., Lawler J. E., 1999, MNRAS, 307, 934

Ruczkowski J., Elantkowska M., Dembczynski J., 2014, J.Q.S.R.T., 145, 20

Russell H. N., Meggers W. F., 1927, Sci. Papers Natl. Bur. Stand. (U.S.), 22, 329

Scott P., Asplund M., Grevesse N., Bergemann M., Sauval A. J., 2015, A\&A, 573, A26

Sikström C. M., Nilsson H., Litzen U., Blom A., Lundberg H., 2002, J. Quant. Spectr. Rad. Transf., 74, 355

Vogel O., Ward L., Arnesen A., Hallin R., Nordling C., Wannstrom A., 1985, Phys. Scr., 31, 166

This paper has been typeset from a $\mathrm{T}_{\mathrm{E}} \mathrm{X} / \mathrm{L} \mathrm{T} \mathrm{E} \mathrm{X}$ file prepared by the author. 